\title{
Volume integral equation for electromagnetic scattering: Rigorous derivation and analysis for a set of multi-layered particles with piecewise-smooth boundaries in a passive host medium
}

\author{
Maxim A. Yurkin ${ }^{1,2, *}$ and Michael I. Mishchenko ${ }^{3}$ \\ ${ }^{1}$ Voevodsky Institute of Chemical Kinetics and Combustion SB RAS, \\ Institutskaya Str. 3, 630090, Novosibirsk, Russia \\ ${ }^{2}$ Novosibirsk State University, Pirogova Str. 2, 630090, Novosibirsk, Russia \\ ${ }^{3}$ NASA Goddard Institute for Space Studies, 2880 Broadway, New York, NY 10025, USA \\ *Corresponding author: yurkin@gmail.com
}

\begin{abstract}
We present a general derivation of the frequency-domain volume integral equation (VIE) for the electric field inside a non-magnetic scattering object from the differential Maxwell equations, transmission boundary conditions, radiation condition at infinity, and locally-finite-energy condition. The derivation applies to an arbitrary spatially finite group of particles made of isotropic materials and embedded in a passive host medium, including those with edges, corners, and intersecting internal interfaces. This is a substantially more general type of scatterer than in all previous derivations. We explicitly treat the strong singularity of the integral kernel, but keep the entire discussion accessible to the applied scattering community. We also consider the known results on the existence and uniqueness of VIE solution and conjecture a general sufficient condition for that. Finally, we discuss an alternative way of deriving the VIE for an arbitrary object by means of a continuous transformation of the everywhere smooth refractive-index function into a discontinuous one. Overall, the paper examines and pushes forward the state-of-the-art understanding of various analytical aspects of the VIE.
\end{abstract}

PACS numbers: 42.25.Fx, 02.70.-c

Submitted to Physical Review A 


\section{INTRODUCTION}

The frequency-domain volume integral equation (VIE) for the electric field inside a non-magnetic scattering object has been known for more than 60 years [1]. It has traditionally been intended to be a rigorous formulation of the electromagnetic-scattering problem equivalent to the more conventional one based on the differential Maxwell equations subject to appropriate boundary conditions [2-4]. Moreover, the VIE has been used as the fundamental basis for a number of "numerically exact" computational methods to simulate electromagnetic scattering, the most popular one being the discrete dipole approximation (DDA) [5]. The latter has been used to calculate electromagnetic scattering by virtually all classes of scatterers, including those with sharp edges and internal interfaces [6-8].

Despite the vast existing literature on the subject (see, e.g., the monographs $[2,4,9]$ ), the theoretical understanding of the VIE remains incomplete and incommensurate to the domain of its actual practical applications. Indeed, the literature is largely grouped around the following two extremes: (i) accessible derivations with all complex issues swept under the rug with the intent to maximally shorten the path to practical computations [4,10,11], and (ii) mathematically rigorous treatises that commence with concepts such as Banach spaces, Hölder continuity, etc. and thus are hardly comprehensible to the applied scattering community [2,9,12-14]. As a consequence, the publications from the first group tend to ignore fundamental issues such as the strong singularity of the integral kernel $[4,10]$ and the explicit use of boundary conditions for a scatterer with a distinct boundary [11], which can potentially lead to ambiguities. On the other hand, rigorous mathematical studies from the second group are typically based on simplified assumptions of smooth particle boundaries and continuous interiors [2,9,12,14], whereas sharp edges/vertices and internal interfaces are hardly mentioned [13]. Moreover, mathematical rigor comes at the expense of various limiting assumptions on the constitutive parameters, e.g., that both the scatterer and the host medium are non-absorbing dielectrics [14] or that the real part of the electric permittivity is positive $[2,12,13]$. As a result, the current understanding of the conditions guarantying the existence and uniqueness of the VIE solution, and that of the scattering problem in general, remains fragmentary, especially in the case of an absorbing host medium (see also Refs. $[3,15,16]$ ). Thus, gray zones exist, where practical numerical simulations have been pursued, but a rigorous mathematical analysis is still not available.

To fill these essential gaps, we present an accessible, self-contained, and general derivation of the VIE from the differential Maxwell equations, transmission boundary conditions, radiation condition at infinity, and locally-finite-energy condition with an explicit treatment of the kernel singularity. Our derivation applies to a representative type of scattering object such as a spatially 
finite group of multi-layered particles with piecewise smooth (intersecting) boundaries and internal interfaces (with a smooth refractive index in between) immersed in a passive unbounded host medium. To further demonstrate the equivalence of the differential and integral formulations of electromagnetic scattering, we derive the former from the latter. We also generalize the results of existing mathematical analyses of the VIE and formulate a conjecture about sufficient conditions ensuring the existence and uniqueness of its solution for this type of scatterer. Finally, we discuss an alternative way of deriving the VIE for an arbitrary object with discontinuities by means of a continuous transformation of the everywhere smooth refractive-index function into a discontinuous one.

\section{FORMULATION OF THE SCATTERING PROBLEM}

Following the lines of derivations in Chapter 4 of Ref. [4] and in Ref. [17], we start with the Maxwell curl equations for time-harmonic [with the implicit $\exp (-\mathrm{i} \omega t)$ convention] electric and magnetic fields, $\mathbf{E}$ and $\mathbf{H}$, assuming non-magnetic isotropic materials throughout the entire space:

$$
\begin{aligned}
& \left.\begin{array}{l}
\nabla \times \mathbf{E}(\mathbf{r})=\mathrm{i} \omega \mu_{0} \mathbf{H}(\mathbf{r}) \\
\nabla \times \mathbf{H}(\mathbf{r})=-\mathrm{i} \omega \varepsilon_{1} \mathbf{E}(\mathbf{r})
\end{array}\right\} \quad \mathbf{r} \in V_{\mathrm{ext}} \\
& \left.\begin{array}{l}
\nabla \times \mathbf{E}(\mathbf{r})=\mathrm{i} \omega \mu_{0} \mathbf{H}(\mathbf{r}) \\
\nabla \times \mathbf{H}(\mathbf{r})=-\mathrm{i} \omega \varepsilon_{2}(\mathbf{r}) \mathbf{E}(\mathbf{r})
\end{array}\right\} \quad \mathbf{r} \in V_{\mathrm{int}}
\end{aligned}
$$

where $\mathrm{i}=\sqrt{-1}, \mu_{0}$ is the vacuum permeability, $V_{\text {ext }}$ is an unbounded homogeneous external medium with a constant electric permittivity $\varepsilon_{1} \neq 0$ and $V_{\text {int }}$ is the interior of a scatterer with a coordinate-dependent permittivity $\varepsilon_{2}$. The entire space is assumed to be devoid of impressed (enforced) sources. Note that, generally, both $\varepsilon_{1}$ and $\varepsilon_{2}$ are complex and depend on the angular frequency $\omega$. In particular, the external medium can be absorbing (lossy), but not active, i.e., we require that $0 \leq \arg \varepsilon_{1}<\pi$ discarding the non-physical option of a negative real $\varepsilon_{1}$ (see Chapter 1 of Ref. [3]).

Equations (1) can be rewritten as a single differential equation:

$$
\begin{gathered}
\nabla \times \nabla \times \mathbf{E}(\mathbf{r})-k_{1}^{2} \mathbf{E}(\mathbf{r})=\mathbf{j}(\mathbf{r}), \quad \mathbf{r} \in \mathbb{R}^{3} \backslash S_{\text {int }}, \\
\mathbf{j}(\mathbf{r}) \stackrel{\text { def }}{=} k_{1}^{2}\left[m^{2}(\mathbf{r})-1\right] \mathbf{E}(\mathbf{r}),
\end{gathered}
$$

where $k_{1}=\omega \sqrt{\varepsilon_{1} \mu_{0}}$ with $\mathfrak{R}\left(k_{1}\right)>0$ and $\mathfrak{I}\left(k_{1}\right) \geq 0$ is the wave number of the exterior, $m(\mathbf{r})$ and $\varepsilon(\mathbf{r})$ are the complex refractive index and permittivity relative to that of the external medium: ${ }^{1}$

$$
m(\mathbf{r})=\sqrt{\varepsilon(\mathbf{r}),} \quad \varepsilon(\mathbf{r}) \stackrel{\text { def }}{=} \begin{cases}1, & \mathbf{r} \in V_{\text {ext }}, \\ \varepsilon_{2}(\mathbf{r}) / \varepsilon_{1}, & \mathbf{r} \in V_{\mathrm{int}}\end{cases}
$$

and $S_{\text {int }}=\partial V_{\text {int }}$. The complex square root in Eq. (4) is potentially ambiguous, but this is not a problem as long as we only use $m^{2}$. More generally, we assume that the support of $m(\mathbf{r})-1$ is

\footnotetext{
${ }^{1}$ In the following discussion we will mostly employ $m$, as that is more common in the light-scattering community.
} 
bounded; $V_{\text {ext }}$ can be defined as the largest unbounded open connected region with $m(\mathbf{r})=1 ; V_{\text {int }}$ is a union of open bounded regions in which $m(\mathbf{r})$ is smooth ${ }^{2}$ [including internal voids with $m(\mathbf{r})=1]$; and $S_{\text {int }}$ is a closed surface containing all discontinuities of $m(\mathbf{r})$ or its derivatives, including the exterior scatterer boundaries and internal interfaces. In particular, we have the following partition in which the components are pair-wise disjoint:

$$
\mathbb{R}^{3}=V_{\text {ext }} \cup V_{\text {int }} \cup S_{\text {int }}
$$

This definition applies to a general finite multi-particle scatterer, with potentially multi-layered components. The particular complex values of $m$ that are physically viable and/or required for wellposedness of the mathematical problem are discussed in Section VII.

An essential further assumption is that $S_{\text {int }}$ consists of several disjoint components:

$$
S_{\text {int }}=\bigcup_{i} S_{i}
$$

each of which is a connected smooth closed surface. Then we have the standard boundary conditions:

$$
\left.\begin{array}{l}
\mathbf{n} \times\left[\mathbf{E}_{1}(\mathbf{r})-\mathbf{E}_{2}(\mathbf{r})\right]=0 \\
\mathbf{n} \times\left[\mathbf{H}_{1}(\mathbf{r})-\mathbf{H}_{2}(\mathbf{r})\right]=0
\end{array}\right\} \mathbf{r} \in S_{\mathrm{int}}
$$

where 1 and 2 label different sides of the specific component of the boundary (the corresponding limits are implied) and $\mathbf{n}$ is the outward-pointing normal to $S_{\text {int }}$.

The total field [i.e., the solution of the inhomogeneous differential equation (2)] can be separated into the incident and scattered fields:

$$
\mathbf{E}(\mathbf{r})=\mathbf{E}_{\mathrm{inc}}(\mathbf{r})+\mathbf{E}_{\mathrm{sca}}(\mathbf{r}) \text {, }
$$

where $\mathbf{E}_{\text {inc }}(\mathbf{r})$ is the solution of the corresponding homogeneous equation [Eq. (2) with a zero righthand side], i.e., the field when no scatterer is present. Mathematically, the latter can be formulated using a Silver-Müller radiation condition [2] to select a single specific solution of Eq. (2),

$$
\mathbf{r} \times\left[\nabla \times \mathbf{E}_{\mathrm{sca}}(\mathbf{r})\right]+\mathrm{i} k_{1} r \mathbf{E}_{\mathrm{sca}}(\mathbf{r}) \underset{r \rightarrow \infty}{\longrightarrow} 0,
$$

uniformly over all directions $\mathbf{r} / r$. Note however that even a weaker condition $\left(L^{2}\right.$-convergence) is sufficient [18]:

$$
\lim _{\Delta \rightarrow \infty} \frac{1}{\Delta^{2}} \oint_{S_{\Delta}} \mathrm{d}^{2} \mathbf{r}\left|\mathbf{r} \times\left[\nabla \times \mathbf{E}_{\mathrm{sca}}(\mathbf{r})\right]+\mathrm{i} k_{1} r \mathbf{E}_{\mathrm{sca}}(\mathbf{r})\right|^{2}=0,
$$

where $S_{\Delta}$ is the spherical surface with a radius $\Delta$ centered at the origin $(\mathbf{r}=\mathbf{n} \Delta)$. The standard scattering problem consists in finding $\mathbf{E}_{\text {sca }}(\mathbf{r})$ satisfying Eqs. (2), (7), (8), and (10) given a physically viable $\mathbf{E}_{\text {inc }}(\mathbf{r})$, i.e., the one satisfying the free-space Maxwell equations.

\footnotetext{
${ }^{2}$ Hereinafter we use "smooth" in the sense "sufficiently smooth" in an effort to keep the discussion relatively simple. However, specific function spaces requiring boundedness or Hölder-continuity of derivatives up to a certain order are discusses in the referenced mathematical literature.
} 


\section{DYADIC GREEN'S FUNCTION AND THEOREM}

Recall now the definition of the free-space dyadic Green's function (Cartesian Green's tensor), e.g., from Appendix B of Ref. [4]:

$$
\overline{\mathbf{G}}\left(\mathbf{r}, \mathbf{r}^{\prime}\right) \stackrel{\text { def }}{=}\left(\overline{\mathbf{I}}+\frac{\nabla \otimes \nabla}{k_{1}^{2}}\right) g\left(\mathbf{r}, \mathbf{r}^{\prime}\right)=\frac{\exp \left(\mathrm{i} k_{1} R\right)}{4 \pi R}\left[\left(\overline{\mathbf{I}}-\frac{\mathbf{R} \otimes \mathbf{R}}{R^{2}}\right)+\frac{\mathrm{i} k_{1} R-1}{k_{1}^{2} R^{2}}\left(\overline{\mathbf{I}}-3 \frac{\mathbf{R} \otimes \mathbf{R}}{R^{2}}\right)\right],
$$

where $\mathbf{R}=\mathbf{r}-\mathbf{r}^{\prime}, R=|\mathbf{R}|, \overline{\mathbf{I}}$ is the unity dyadic, and $g\left(\mathbf{r}, \mathbf{r}^{\prime}\right)$ is the scalar Green's function:

$$
g\left(\mathbf{r}, \mathbf{r}^{\prime}\right)=g(R) \stackrel{\text { def }}{=} \frac{\exp \left(\mathrm{i} k_{1} R\right)}{4 \pi R}
$$

satisfying

$$
\left(\nabla^{2}+k_{1}^{2}\right) g\left(\mathbf{r}, \mathbf{r}^{\prime}\right)=-\delta\left(\mathbf{r}-\mathbf{r}^{\prime}\right),
$$

where $\delta(\mathbf{R})$ is the tree-dimensional delta function.

The essential property of the Green's dyadic is that it satisfies

$$
\nabla \times \nabla \times \overline{\mathbf{G}}\left(\mathbf{r}, \mathbf{r}^{\prime}\right)-k_{1}^{2} \overline{\mathbf{G}}\left(\mathbf{r}, \mathbf{r}^{\prime}\right)=\overline{\mathbf{I}} \delta\left(\mathbf{r}-\mathbf{r}^{\prime}\right) .
$$

Also, it is symmetric with respect to both argument interchange and dyadic transposition (superscript T),

$$
\overline{\mathbf{G}}\left(\mathbf{r}^{\prime}, \mathbf{r}\right)=\overline{\mathbf{G}}\left(\mathbf{r}, \mathbf{r}^{\prime}\right)=\left[\overline{\mathbf{G}}\left(\mathbf{r}, \mathbf{r}^{\prime}\right)\right]^{\mathrm{T}},
$$

and satisfies the following radiation condition [cf. Eq. (9)]:

$$
\mathbf{r} \times[\nabla \times \overline{\mathbf{G}}(\mathbf{r}, \mathbf{0})]+\mathrm{i} k_{1} r \overline{\mathbf{G}}(\mathbf{r}, \mathbf{0}) \underset{r \rightarrow \infty}{\longrightarrow} \mathcal{O}(1 / r) .
$$

In particular, it converges to zero uniformly over all directions $\mathbf{r} / r$. In the limit of a very small $R$,

$$
\overline{\mathbf{G}}\left(\mathbf{r}, \mathbf{r}^{\prime}\right) \underset{R \rightarrow 0}{\longrightarrow} \overline{\mathbf{G}}_{\mathrm{st}}\left(\mathbf{r}, \mathbf{r}^{\prime}\right)+\mathcal{O}(1 / R),
$$

where the static Green's dyadic is

$$
\overline{\mathbf{G}}_{\mathrm{st}}\left(\mathbf{r}, \mathbf{r}^{\prime}\right)=(\nabla \otimes \nabla) \frac{1}{4 \pi k_{1}^{2} R}=-\frac{1}{4 \pi k_{1}^{2} R^{3}}\left(\overline{\mathbf{I}}-3 \frac{\mathbf{R} \otimes \mathbf{R}}{R^{2}}\right) .
$$

Moreover,

$$
\nabla \times \overline{\mathbf{G}}\left(\mathbf{r}, \mathbf{r}^{\prime}\right)=(\nabla g) \times \overline{\mathbf{I}}=\frac{\mathbf{R} \times \overline{\mathbf{I}}}{4 \pi R^{3}} \exp \left(\mathrm{i} k_{1} R\right)\left(\mathrm{i} k_{1} R-1\right) \underset{R \rightarrow 0}{\longrightarrow}-\frac{\mathbf{R} \times \overline{\mathbf{I}}}{4 \pi R^{3}}+\mathcal{O}(1) .
$$

The Green's dyadic and Eq. (14) are commonly used together with the dyadic Green's theorem (Eq. (A4.75) of Ref. [9]):

$$
\int_{V} \mathrm{~d}^{3} \mathbf{r}[(\nabla \times \nabla \times \mathbf{a}) \cdot \overline{\mathbf{A}}-\mathbf{a} \cdot(\nabla \times \nabla \times \overline{\mathbf{A}})]=\oint_{\partial V} \mathrm{~d}^{2} \mathbf{r}\{(\mathbf{n} \times \mathbf{a}) \cdot(\nabla \times \overline{\mathbf{A}})+[\mathbf{n} \times(\nabla \times \mathbf{a})] \cdot \overline{\mathbf{A}}\},
$$

where $V$ is any bounded region, $\mathbf{n}$ is the outward surface normal, and $\mathbf{a}$ and $\overline{\mathbf{A}}$ are an arbitrary vector and a dyadic varying smoothly with $\mathbf{r}$ (i.e., at least, having integrable second derivatives). 


\section{DERIVATION OF THE VIE FOR A SIMPLE SCATTERER}

We first consider a simple single-body scatterer $V_{\text {int }}$ without internal interfaces, i.e., the one having a connected boundary $S_{\text {int }}$ (or, equivalently, connected $V_{\text {int }}$, see Fig. 1). Proceeding along the lines of Chapter 4.3 of Ref. [4] (but with an interchange of $\mathbf{r}$ and $\mathbf{r}^{\prime}$ ), we scalar post-multiply Eq. (2) by $\overline{\mathbf{G}}\left(\mathbf{r}, \mathbf{r}^{\prime}\right)$ and scalar pre-multiply Eq. (14) by $\mathbf{E}_{\text {sca }}(\mathbf{r})$ :

$$
\begin{gathered}
{\left[\nabla \times \nabla \times \mathbf{E}_{\mathrm{sca}}(\mathbf{r})\right] \cdot \overline{\mathbf{G}}\left(\mathbf{r}, \mathbf{r}^{\prime}\right)-k_{1}^{2} \mathbf{E}_{\mathrm{sca}}(\mathbf{r}) \cdot \overline{\mathbf{G}}\left(\mathbf{r}, \mathbf{r}^{\prime}\right)=\mathbf{j}(\mathbf{r}) \cdot \overline{\mathbf{G}}\left(\mathbf{r}, \mathbf{r}^{\prime}\right),} \\
\mathbf{E}_{\mathrm{sca}}(\mathbf{r}) \cdot\left[\nabla \times \nabla \times \overline{\mathbf{G}}\left(\mathbf{r}, \mathbf{r}^{\prime}\right)\right]-k_{1}^{2} \mathbf{E}_{\mathrm{sca}}(\mathbf{r}) \cdot \overline{\mathbf{G}}\left(\mathbf{r}, \mathbf{r}^{\prime}\right)=\mathbf{E}_{\mathrm{sca}}(\mathbf{r}) \delta\left(\mathbf{r}-\mathbf{r}^{\prime}\right) .
\end{gathered}
$$

Subtracting Eq. (22) from Eq. (21) yields

$$
\begin{gathered}
{\left[\nabla \times \nabla \times \mathbf{E}_{\mathrm{sca}}(\mathbf{r})\right] \cdot \overline{\mathbf{G}}\left(\mathbf{r}, \mathbf{r}^{\prime}\right)-\mathbf{E}_{\mathrm{sca}}(\mathbf{r}) \cdot\left[\nabla \times \nabla \times \overline{\mathbf{G}}\left(\mathbf{r}, \mathbf{r}^{\prime}\right)\right]} \\
=\mathbf{j}(\mathbf{r}) \cdot \overline{\mathbf{G}}\left(\mathbf{r}, \mathbf{r}^{\prime}\right)-\mathbf{E}_{\mathrm{sca}}(\mathbf{r}) \delta\left(\mathbf{r}-\mathbf{r}^{\prime}\right) .
\end{gathered}
$$

A typical derivation of the VIE $[4,10]$ would use Eq. (20) with $\mathbf{a}=\mathbf{E}_{\text {sca }},{ }^{3} \overline{\mathbf{A}}=\overline{\mathbf{G}}$, and $V=V_{\text {int }}$, to be further simplified using Eq. (23). However, this path is not completely rigorous (leads to ambiguous results) due to the strong singularity of $\overline{\mathbf{G}}\left(\mathbf{r}, \mathbf{r}^{\prime}\right)$ at $\mathbf{r}=\mathbf{r}^{\prime}$ [11]. Two different ways to address this problem are mentioned in the footnote on p. 98 of Ref. [9]: rigorous treatment of delta functions and their derivatives or exclusion of the singularity.

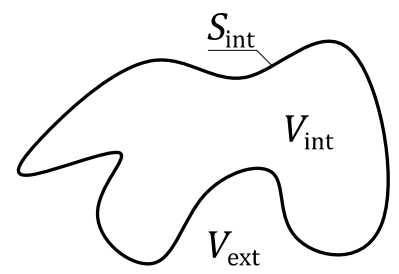

Fig. 1. A simple single-body scatterer with a smooth boundary and without internal interfaces.

We choose the second option and define $V_{\delta}$ (and $S_{\delta}=\partial V_{\delta}$ ) as a small volume around $\mathbf{r}^{\prime}$ which shrinks to $\mathbf{r}^{\prime}$ when $\delta \rightarrow 0$ while keeping the same shape. ${ }^{4}$ Then, we can apply the above described combination of Eqs. (20) and (23) to $V=V_{\text {int }} \backslash V_{\delta}$ (assuming $\mathbf{r}^{\prime} \in V_{\text {int }}$ ) and let $\delta \rightarrow 0$ :

$$
\begin{aligned}
& \lim _{\delta \rightarrow 0} \int_{V_{\mathrm{int}} \backslash V_{\delta}} \mathrm{d}^{3} \mathbf{r}\left[\mathbf{j}(\mathbf{r}) \cdot \overline{\mathbf{G}}\left(\mathbf{r}, \mathbf{r}^{\prime}\right)-\mathbf{E}_{\mathrm{sca}}(\mathbf{r}) \delta\left(\mathbf{r}-\mathbf{r}^{\prime}\right)\right] \\
& =\left(\oint_{S_{\mathrm{int}}}-\lim _{\delta \rightarrow 0} \oint_{S_{\delta}}\right) \mathrm{d}^{2} \mathbf{r}\left\{\left(\mathbf{n} \times \mathbf{E}_{\mathrm{sca}}(\mathbf{r})\right) \cdot\left(\nabla \times \overline{\mathbf{G}}\left(\mathbf{r}, \mathbf{r}^{\prime}\right)\right)+\left[\mathbf{n} \times\left(\nabla \times \mathbf{E}_{\mathrm{sca}}(\mathbf{r})\right)\right] \cdot \overline{\mathbf{G}}\left(\mathbf{r}, \mathbf{r}^{\prime}\right)\right\} .
\end{aligned}
$$

If $\mathbf{r}^{\prime} \in V_{\text {ext }}$, no exclusion of the singularity is needed and Eq. (24) is valid with all the $\delta$-related parts being removed (as discussed below).

The $\delta$-function term on the left-hand side of Eq. (24) always vanishes, which may seem counterintuitive, but it would be compensated by the additional terms on the right-hand side. To

\footnotetext{
${ }^{3}$ Smoothness of $\mathbf{E}_{\text {sca }}(\mathbf{r})$ follows from smoothness of $m(\mathbf{r})$, which is further discussed in Section IX.

${ }^{4}$ This $\delta$ should not be confused with the delta function.
} 
evaluate the first term in the integral over $S_{\delta}$, we use Eq. (19). Since the latter has a $R^{-2}$ singularity, we need to keep only the zeroth order of $\mathbf{E}_{\text {sca }}(\mathbf{r})$, which is $\mathbf{E}_{\mathrm{sca}}\left(\mathbf{r}^{\prime}\right)$. Thus,

$$
\begin{aligned}
\lim _{\delta \rightarrow 0} \oint_{S_{\delta}} \mathrm{d}^{2} \mathbf{r}\left(\mathbf{n} \times \mathbf{E}_{\mathrm{sca}}(\mathbf{r})\right) \cdot\left(\nabla \times \overline{\mathbf{G}}\left(\mathbf{r}, \mathbf{r}^{\prime}\right)\right)=-\mathbf{E}_{\mathrm{sca}}\left(\mathbf{r}^{\prime}\right) \cdot \lim _{\delta \rightarrow 0} \oint_{S_{\delta}} \mathrm{d}^{2} \mathbf{r}\left[\mathbf{n} \times\left(\nabla \times \overline{\mathbf{G}}\left(\mathbf{r}, \mathbf{r}^{\prime}\right)\right)\right] \\
=\mathbf{E}_{\mathrm{sca}}\left(\mathbf{r}^{\prime}\right) \cdot \lim _{\delta \rightarrow 0} \oint_{S_{\delta}} \mathrm{d}^{2} \mathbf{r} \frac{\mathbf{n} \times(\mathbf{R} \times \overline{\mathbf{I}})}{4 \pi R^{3}}=\mathbf{E}_{\mathrm{sca}}\left(\mathbf{r}^{\prime}\right) \cdot\left(\oint_{S_{\delta}} \mathrm{d}^{2} \mathbf{r} \frac{\mathbf{R} \otimes \mathbf{n}}{4 \pi R^{3}}-\overline{\mathbf{I}} \operatorname{tr}(\overline{\mathbf{L}})\right) \\
=(\overline{\mathbf{L}}-\overline{\mathbf{I}}) \cdot \mathbf{E}_{\mathrm{sca}}\left(\mathbf{r}^{\prime}\right),
\end{aligned}
$$

where the first and third transformations are based on the dyadic identities

$$
(\mathbf{a} \times \mathbf{b}) \cdot \overline{\mathbf{A}}=\mathbf{a} \cdot(\mathbf{b} \times \overline{\mathbf{A}}) \text { and } \mathbf{a} \times(\mathbf{b} \times \overline{\mathbf{A}})=\mathbf{b} \otimes(\mathbf{a} \cdot \overline{\mathbf{A}})-\overline{\mathbf{A}}(\mathbf{a} \cdot \mathbf{b}),
$$

respectively, while

$$
\overline{\mathbf{L}} \stackrel{\text { def }}{=} \oint_{S_{\delta}} \mathrm{d}^{2} \mathbf{r} \frac{\mathbf{n} \otimes \mathbf{R}}{4 \pi R^{3}}
$$

is the self-term dyadic (the source term) [11] (a.k.a. depolarization dyadic - see Chapter 3.9 of Ref. [9]) which depends on the shape but not on the size of $S_{\delta}$ (the limit in the fourth part of Eq. (27) was removed accordingly) and on the position of $\mathbf{r}^{\prime}$ inside $S_{\delta}$. Importantly, this dyadic is always real symmetric with $\operatorname{tr}(\overline{\mathbf{L}})=1$ (hence the last transformation in Eq. (25)). For a sphere or a cube centered at $\mathbf{r}^{\prime}$, it takes the simplest form $\overline{\mathbf{L}}=\overline{\mathbf{I}} / 3$ [11].

In the second term of the integral over $S_{\delta}$ in Eq. (24), $\overline{\mathbf{G}}\left(\mathbf{r}, \mathbf{r}^{\prime}\right)$ can be replaced by $\overline{\mathbf{G}}_{\mathrm{st}}\left(\mathbf{r}, \mathbf{r}^{\prime}\right)$ [cf. Eq. (17)]. Then

$$
\begin{aligned}
\lim _{\delta \rightarrow 0} \oint_{S_{\delta}} \mathrm{d}^{2} \mathbf{r}[\mathbf{n} \times & \left.\left(\nabla \times \mathbf{E}_{\mathrm{sca}}(\mathbf{r})\right)\right] \cdot \overline{\mathbf{G}}\left(\mathbf{r}, \mathbf{r}^{\prime}\right) \\
= & -\frac{1}{k_{1}^{2}} \lim _{\delta \rightarrow 0} \oint_{S_{\delta}} \mathrm{d}^{2} \mathbf{r}\left[\mathbf{n} \times\left(\nabla \times \mathbf{E}_{\mathrm{sca}}(\mathbf{r})\right)\right] \cdot\left(\nabla \otimes \frac{\mathbf{R}}{4 \pi R^{3}}\right) \\
= & -\frac{1}{k_{1}^{2}} \lim _{\delta \rightarrow 0} \oint_{S_{\delta}} \mathrm{d}^{2} \mathbf{r}\left(\nabla \times \nabla \times \mathbf{E}_{\mathrm{sca}}(\mathbf{r})\right) \cdot \frac{\mathbf{n} \otimes \mathbf{R}}{4 \pi R^{3}}=-\overline{\mathbf{L}} \cdot\left(\frac{\mathbf{j}\left(\mathbf{r}^{\prime}\right)}{k_{1}^{2}}+\mathbf{E}_{\mathrm{sca}}\left(\mathbf{r}^{\prime}\right)\right),
\end{aligned}
$$

where the last transformation keeps only the zeroth order of $\nabla \times \nabla \times \mathbf{E}_{\mathrm{sca}}(\mathbf{r})$ and uses Eqs. (2) and (27), while the second one is based on

$$
\begin{aligned}
& \oint_{S} \mathrm{~d}^{2} \mathbf{r}(\mathbf{n} \times \mathbf{a}) \cdot(\nabla \otimes \mathbf{b})=\oint_{S} \mathrm{~d}^{2} \mathbf{r} \mathbf{n} \cdot[\mathbf{a} \times(\nabla \otimes \mathbf{b})] \\
& =-\underbrace{\oint_{S} \mathrm{~d}^{2} \mathbf{r} \mathbf{n} \cdot[\nabla \times(\mathbf{a} \otimes \mathbf{b})]}_{\overline{\mathbf{0}}}+\oint_{S} \mathrm{~d}^{2} \mathbf{r} \mathbf{n} \cdot[(\nabla \times \mathbf{a}) \otimes \mathbf{b}]=\oint_{S} \mathrm{~d}^{2} \mathbf{r}(\nabla \times \mathbf{a}) \cdot(\mathbf{n} \otimes \mathbf{b}),
\end{aligned}
$$

where $\overline{\mathbf{0}}$ is a zero dyadic, $\mathbf{a}(\mathbf{r})$ and $\mathbf{b}(\mathbf{r})$ are arbitrary smooth on $S$ vector fields [in our case $\nabla \times$ $\mathbf{E}_{\text {sca }}(\mathbf{r})$ and $\mathbf{R} /\left(4 \pi R^{3}\right)$, respectively], and the zeroing of the term in the middle follows from the 
dyadic version of the Stokes theorem (Eq. (A4.70) of Ref. [9]) and the absence of a boundary for a closed surface.

Before finalizing this derivation, let us define an auxiliary vector function based on the righthand side of Eq. (24):

$$
\mathbf{X}\left(\mathbf{r}, \mathbf{r}^{\prime}\right) \stackrel{\text { def }}{=}\left(\mathbf{n} \times \mathbf{E}_{\mathrm{sca}}(\mathbf{r})\right) \cdot\left(\nabla \times \overline{\mathbf{G}}\left(\mathbf{r}, \mathbf{r}^{\prime}\right)\right)+\left[\mathbf{n} \times\left(\nabla \times \mathbf{E}_{\mathrm{sca}}(\mathbf{r})\right)\right] \cdot \overline{\mathbf{G}}\left(\mathbf{r}, \mathbf{r}^{\prime}\right),
$$

which also implicitly depends on the normal $\mathbf{n}$ of the integration surface $S$. Importantly, $\mathbf{X}\left(\mathbf{r}, \mathbf{r}^{\prime}\right)$ is continuous when $\mathbf{r}$ crosses $S$ (if $\mathbf{r}^{\prime} \notin S$ ), owing to either the boundary conditions (7) (and $\nabla \times \mathbf{E}=$ $\left.\mathrm{i} \omega \mu_{0} \mathbf{H}\right)$ or the continuity of all constituent functions if $m(\mathbf{r})$ is continuous across $S$. Thus, it does not matter which side of the surface the $\mathbf{X}\left(\mathbf{r}, \mathbf{r}^{\prime}\right)$ is integrated over, as long as the same normal is used. In the following, we always assume the outward normal $\mathbf{n}$ to any closed surface (considered individually), and the orientation of this normal with respect to an integration volume is accounted for by a sign before the surface integral, as in Eq. (24).

Another property of $\mathbf{X}\left(\mathbf{r}, \mathbf{r}^{\prime}\right)$ is

$$
\lim _{\Delta \rightarrow \infty} \oint_{S_{\Delta}} d^{2} \mathbf{r} \mathbf{X}(\mathbf{r}, \mathbf{0})=\mathbf{0}
$$

which follows from Eq. (10) [18], as briefly discussed in the following. First, Lemma 2 of Ref. [18] states that

$$
\oint_{S_{\Delta}} \mathrm{d} \Omega\left|r \mathbf{E}_{\mathrm{sca}}(\mathbf{r})\right|^{2}=\mathcal{O}(1)
$$

Second,

$$
\begin{aligned}
\oint_{S_{\Delta}} \mathrm{d}^{2} \mathbf{r} \mathbf{X}(\mathbf{r}, \mathbf{0}) & =\oint_{S_{\Delta}} \mathrm{d} \Omega\left\{\mathbf{r} \times\left[\nabla \times \mathbf{E}_{\mathrm{sca}}(\mathbf{r})\right]+\mathrm{i} k_{1} r \mathbf{E}_{\mathrm{sca}}(\mathbf{r})\right\} \cdot r \overline{\mathbf{G}}(\mathbf{r}, \mathbf{0}) \\
& -\oint_{S_{\Delta}} \mathrm{d} \Omega r \mathbf{E}_{\mathrm{sca}}(\mathbf{r}) \cdot\left\{\mathbf{r} \times[\nabla \times \overline{\mathbf{G}}(\mathbf{r}, \mathbf{0})]+\mathrm{i} k_{1} r \overline{\mathbf{G}}(\mathbf{r}, \mathbf{0})\right\} .
\end{aligned}
$$

Third, applying the Cauchy-Schwarz inequality to Eq. (33) and using Eqs. (10), (11), (16), and (32), we obtain Eq. (31). Note that nonzero absorption in the external medium would only make the derivation easier, since it causes $\overline{\mathbf{G}}(\mathbf{r}, \mathbf{0})$ to decay exponentially when $r \rightarrow \infty$.

Next, we combine Eqs. (24), (25), and (28) and interchange $\mathbf{r}$ and $\mathbf{r}^{\prime}$ to shift the focus from the integration variable and conform to a common notation [4]. This yields

$$
\lim _{\delta \rightarrow 0} \int_{V_{\mathrm{int}} \backslash V_{\delta}} \mathrm{d}^{3} \mathbf{r}^{\prime} \mathbf{j}\left(\mathbf{r}^{\prime}\right) \cdot \overline{\mathbf{G}}\left(\mathbf{r}^{\prime}, \mathbf{r}\right)-\chi_{V_{\mathrm{int}}}(\mathbf{r})\left[\frac{\overline{\mathbf{L}} \cdot \mathbf{j}(\mathbf{r})}{k_{1}^{2}}+\mathbf{E}_{\mathrm{sca}}(\mathbf{r})\right]=\oint_{S_{\mathrm{int}}} \mathrm{d}^{2} \mathbf{r}^{\prime} \mathbf{X}\left(\mathbf{r}^{\prime}, \mathbf{r}\right),
$$

where

$$
\chi_{V}(\mathbf{r}) \stackrel{\text { def }}{=} \begin{cases}1, & \mathbf{r} \in V \\ 0, & \text { otherwise }\end{cases}
$$


is the indicator function which allows for a single expression for both the interior and the exterior of the scatterer. For the latter, Eq. (34) follows directly from Eq. (24) with the $\delta$-related parts being removed.

Repeating the derivation starting from Eq. (24) for $V_{\text {ext }}$ instead of $V_{\text {int }}$ and assuming $\partial V_{\text {ext }}=$ $S_{\Delta} \cup S_{\text {int }}^{-}$, where the superscript "-" denotes the non-standard direction of the normal, we obtain:

$$
\begin{gathered}
\lim _{\delta \rightarrow 0} \int_{V_{\text {ext }} \backslash V_{\delta}} \mathrm{d}^{3} \mathbf{r}^{\prime} \mathbf{j}\left(\mathbf{r}^{\prime}\right) \cdot \overline{\mathbf{G}}\left(\mathbf{r}^{\prime}, \mathbf{r}\right)-\chi_{V_{\mathrm{ext}}}(\mathbf{r})\left[\frac{\overline{\mathbf{L}} \cdot \mathbf{j}(\mathbf{r})}{k_{1}^{2}}+\mathbf{E}_{\mathrm{sca}}(\mathbf{r})\right] \\
=\left(\lim _{\Delta \rightarrow \infty} \oint_{S_{\Delta}}-\oint_{S_{\text {int }}}\right) \mathrm{d}^{2} \mathbf{r}^{\prime} \mathbf{X}\left(\mathbf{r}^{\prime}, \mathbf{r}\right) .
\end{gathered}
$$

Adding up Eqs. (34) and (36) and employing Eqs. (3), (4), (15), and (31) along with $\chi_{V_{\text {ext }}}(\mathbf{r})+$ $\chi_{V_{\text {int }}}(\mathbf{r}) \equiv 1\left(\mathbf{r} \in \mathbb{R}^{3} \backslash S_{\text {int }}\right)$ yields

$$
\mathbf{E}_{\text {sca }}(\mathbf{r})=\lim _{\delta \rightarrow 0} \int_{V_{\text {int }} \backslash V_{\delta}} \mathrm{d}^{3} \mathbf{r}^{\prime} \overline{\mathbf{G}}\left(\mathbf{r}, \mathbf{r}^{\prime}\right) \cdot \mathbf{j}\left(\mathbf{r}^{\prime}\right)-\frac{\overline{\mathbf{L}} \cdot \mathbf{j}(\mathbf{r})}{k_{1}^{2}}
$$

or equivalently

$$
\mathbf{E}(\mathbf{r})=\mathbf{E}_{\text {inc }}(\mathbf{r})+k_{1}^{2} \lim _{\delta \rightarrow 0} \int_{V_{\text {int }} \backslash V_{\delta}} \mathrm{d}^{3} \mathbf{r}^{\prime}\left[m^{2}\left(\mathbf{r}^{\prime}\right)-1\right] \overline{\mathbf{G}}\left(\mathbf{r}, \mathbf{r}^{\prime}\right) \cdot \mathbf{E}\left(\mathbf{r}^{\prime}\right)-\left[m^{2}(\mathbf{r})-1\right] \overline{\mathbf{L}} \cdot \mathbf{E}(\mathbf{r}),
$$

where $\mathbf{r} \in \mathbb{R}^{3} \backslash S_{\text {int }}$ and $V_{\text {int }}$ in the integrals can be replaced by $\mathbb{R}^{3}$ (assuming that $S_{\text {int }}$ has a zero volume and is isolated in the actual integration). Note also that Eqs. (37) and (38) hold for $\mathbf{r} \in \mathbb{R}^{3}$ when $m(\mathbf{r})$ is smooth in $\mathbb{R}^{3}$ but $m^{2}(\mathbf{r})-1$ has a finite support; the latter effectively replaces $V_{\text {int }}$.

The main advantage of the above derivation in comparison with the more common ones $[2,4]$ is that it treats the singularity of $\overline{\mathbf{G}}$ rigorously and explicitly. In this respect it is similar to the work by Yaghjian [11] and van Bladel (Chapter 7.9 of Ref. [9]), but applies to the general boundary conditions (7) and is more explicit in the employment of the radiation condition (10). The importance of a careful treatment of the singularity was discussed in detail in Ref. [11]. More recently, it was further exemplified by the use of rectangular cuboid elementary volumes (dipoles) in the DDA [19]. In this case the non-symmetric shape of $S_{\delta}$ implicitly leads to ambiguities which can only be settled through an explicit consideration.

We additionally note that the strong singularity can be avoided by considering a different integro-differential equation, see, e.g., Chapter 12.5.2 of Ref. [9]:

$$
\mathbf{E}(\mathbf{r})=\mathbf{E}_{\text {inc }}(\mathbf{r})+\left(k_{1}^{2} \overline{\mathbf{I}}+\nabla \otimes \nabla\right) \cdot \int_{V_{\text {int }}} \mathrm{d}^{3} \mathbf{r}^{\prime}\left[m^{2}\left(\mathbf{r}^{\prime}\right)-1\right] g\left(\mathbf{r}, \mathbf{r}^{\prime}\right) \mathbf{E}\left(\mathbf{r}^{\prime}\right) .
$$

As noted in Ref. [11], the exclusion of $V_{\delta}$ and the $L$-term in Eq. (38) appear exactly from the proper interchange of differentiation and integration in Eq. (39) [cf. Eq. (11)]. Since Eq. (39) is less 
commonly used as a basis of numerical methods, we do not discuss it in detail. Most importantly, the equivalence of Eqs. (38) and (39) holds as long as $\mathbf{E}(\mathbf{r})$ is smooth in $V_{\text {int }}$.

\section{EXTENSION TO A SET OF SEVERAL MULTI-LAYERED PARTICLES}

We further consider a scatterer partitioned into several disjoint components,

$$
V_{\text {int }}=\bigcup_{j} V_{j}
$$

each of which is a connected open region with a smooth $m(\mathbf{r})$. Then Eq. (6) implies that each $S_{i}$ separates exactly two regions from the list $V_{\text {ext }}, V_{1}, V_{2}, \ldots$ and contributes to their boundaries with opposite signs of the surface normal (Fig. 2).

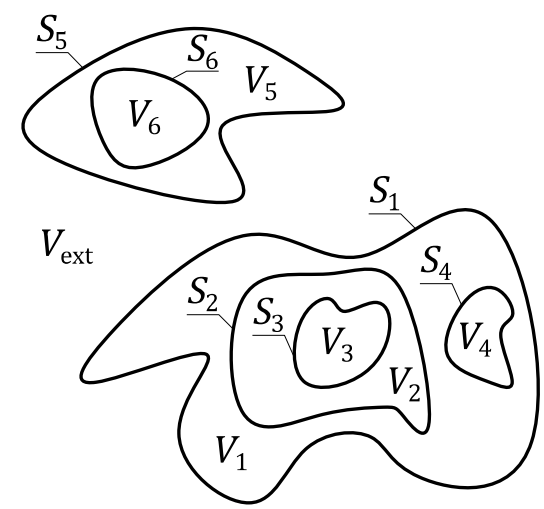

Fig. 2. An example of a complex multi-body multi-layered scatterer with smooth interfaces. Each closed surface separates exactly two domains, one of which may be the external medium.

Using the results obtained in the preceding sections, it is trivial to generalize the VIE to such a scatterer. We write down Eq. (34) for each $V_{j}$ and add them up together with Eq. (36). Then the integrals over each of the $S_{i}$ will occur exactly twice on the right-hand side and with opposite signs and thereby will cancel each other. The left-hand side will lead exactly to Eq. (37) if one makes use of

$$
\chi_{V_{\mathrm{ext}}}(\mathbf{r})+\sum_{j} \chi_{V_{j}}(\mathbf{r})=1-\chi_{s_{\mathrm{int}}}(\mathbf{r}),
$$

which follows from Eqs. (5) and (40) as well as from $\chi_{V}(\mathbf{r})$ being additive with respect to the union of disjoint sets. The possibility of such an extension was mentioned in Chapter VI of Müller [2], but we are unaware of any previous explicit derivation.

\section{EQUIVALENCE OF VIE AND DIFFERENTIAL EQUATIONS}

The goal of this section is to prove the equivalence of the boundary-value problem for the differential Maxell equations and the VIE by explicitly deriving Eq. (2), the boundary conditions 
(7), and the radiation condition (9) from Eq. (38). In principle, one can analyze the invertibility of all intermediate steps in the previous sections, but a direct derivation is both simpler and more instructive.

The derivation of Eq. (2) is completely trivial for $\mathbf{r} \in V_{\text {ext }}$, where the third term (with $\overline{\mathbf{L}}$ ) in Eq. (38) vanishes and $V_{\delta}$ can be removed from the integral since $\overline{\mathbf{G}}\left(\mathbf{r}, \mathbf{r}^{\prime}\right)$ does not have singularities in the integration domain $\left(\mathbf{r}^{\prime} \in V_{\text {int }}\right)$. Moreover, differentiation and integration can be freely interchanged, leading to

$$
\begin{aligned}
& \nabla \times \nabla \times \mathbf{E}(\mathbf{r})-k_{1}^{2} \mathbf{E}(\mathbf{r})=\nabla \times \nabla \times \mathbf{E}_{\mathrm{inc}}(\mathbf{r})-k_{1}^{2} \mathbf{E}_{\mathrm{inc}}(\mathbf{r}) \\
& +\int_{V_{\mathrm{int}}} \mathrm{d}^{3} \mathbf{r}^{\prime}\left[\nabla \times \nabla \times \overline{\mathbf{G}}\left(\mathbf{r}, \mathbf{r}^{\prime}\right)-k_{1}^{2} \overline{\mathbf{G}}\left(\mathbf{r}, \mathbf{r}^{\prime}\right)\right] \cdot \mathbf{j}\left(\mathbf{r}^{\prime}\right)=\mathbf{0},
\end{aligned}
$$

where we have used the homogeneous version of Eq. (2) for $\mathbf{E}_{\text {inc }}(\mathbf{r})$ and Eq. (14).

For $\mathbf{r} \in V_{\text {int }}$ the situation is more complicated owing to the fact that the exclusion volume $V_{\delta}$ depends explicitly on the differentiation variable $\mathbf{r}$ upon which $\nabla \times \nabla \times$ is acting. Fortunately, this dependence is simple (amounts to a translation); hence, the Reynolds transport theorem (or multidimensional Leibnitz integral rule) implies

$$
\nabla \times \int_{V_{\mathrm{int}} \backslash V_{\delta}(\mathbf{r})} \mathrm{d}^{3} \mathbf{r}^{\prime} \mathbf{a}\left(\mathbf{r}, \mathbf{r}^{\prime}\right)=\int_{V_{\mathrm{int}} \backslash V_{\delta}(\mathbf{r})} \mathrm{d}^{3} \mathbf{r}^{\prime}\left[\nabla \times \mathbf{a}\left(\mathbf{r}, \mathbf{r}^{\prime}\right)\right]-\oint_{S_{\delta}(\mathbf{r})} \mathrm{d}^{2} \mathbf{r}^{\prime}\left[\mathbf{n} \times \mathbf{a}\left(\mathbf{r}, \mathbf{r}^{\prime}\right)\right],
$$

where the minus sign appears due to the natural normal to $V_{\text {int }} \backslash V_{\delta}$ pointing inside $S_{\delta}$ (in contrast to the used n). Next, Eq. (38) itself implies that the limit $\delta \rightarrow 0$ is well-behaved (smooth over r); hence, it can be interchanged with differentiation, which together with Eq. (43) implies

$$
\begin{aligned}
& \nabla \times \lim _{\delta \rightarrow 0} \int_{V_{\text {int }} \backslash V_{\delta}} \mathrm{d}^{3} \mathbf{r}^{\prime} \overline{\mathbf{G}}\left(\mathbf{r}, \mathbf{r}^{\prime}\right) \cdot \mathbf{j}\left(\mathbf{r}^{\prime}\right) \\
& \quad=\lim _{\delta \rightarrow 0} \int_{V_{\text {int }} \backslash V_{\delta}} \mathrm{d}^{3} \mathbf{r}^{\prime}\left[\nabla \times \overline{\mathbf{G}}\left(\mathbf{r}, \mathbf{r}^{\prime}\right)\right] \cdot \mathbf{j}\left(\mathbf{r}^{\prime}\right)-\lim _{\delta \rightarrow 0} \oint_{S_{\delta}} \mathrm{d}^{2} \mathbf{r}^{\prime}\left[\mathbf{n} \times\left(\overline{\mathbf{G}}\left(\mathbf{r}, \mathbf{r}^{\prime}\right) \cdot \mathbf{j}\left(\mathbf{r}^{\prime}\right)\right)\right] .
\end{aligned}
$$

The surface integral is transformed similarly to Eqs. (28) and (29). $\overline{\mathbf{G}}\left(\mathbf{r}, \mathbf{r}^{\prime}\right)$ can be replaced by $\overline{\mathbf{G}}_{\mathrm{st}}\left(\mathbf{r}, \mathbf{r}^{\prime}\right)$ [cf. Eq. (17)], leading to

$$
\begin{aligned}
\lim _{\delta \rightarrow 0} \oint_{S_{\delta}} \mathrm{d}^{2} \mathbf{r}^{\prime}[\mathbf{n} & \left.\times\left(\overline{\mathbf{G}}\left(\mathbf{r}, \mathbf{r}^{\prime}\right) \cdot \mathbf{j}\left(\mathbf{r}^{\prime}\right)\right)\right]=-\frac{1}{k_{1}^{2}} \lim _{\delta \rightarrow 0} \oint_{S_{\delta}} \mathrm{d}^{2} \mathbf{r}^{\prime}\left[\mathbf{n} \times\left(\left(\nabla^{\prime} \otimes \frac{\mathbf{R}^{\prime}}{4 \pi R^{3}}\right) \cdot \mathbf{j}\left(\mathbf{r}^{\prime}\right)\right)\right] \\
& =-\frac{1}{k_{1}^{2}} \lim _{\delta \rightarrow 0} \oint_{S_{\delta}} \mathrm{d}^{2} \mathbf{r}^{\prime}\left[\nabla^{\prime}{ }_{\mathbf{j}} \times\left(\frac{\mathbf{n} \otimes \mathbf{R}^{\prime}}{4 \pi R^{3}} \cdot \mathbf{j}\left(\mathbf{r}^{\prime}\right)\right)\right]=-\nabla \times\left[\frac{\overline{\mathbf{L}} \cdot \mathbf{j}(\mathbf{r})}{k_{1}^{2}}\right],
\end{aligned}
$$

where $\mathbf{R}^{\prime}=\mathbf{r}^{\prime}-\mathbf{r}=-\mathbf{R}$; the subscript $\mathbf{j}$ indicates that $\nabla$ acts only on the corresponding function to the right of it; the last transformation follows from Eq. (27) and leaves only the zeroth order of derivatives of $\mathbf{j}\left(\mathbf{r}^{\prime}\right)$; and the middle transformation is based on 


$$
\begin{aligned}
\oint_{S} \mathrm{~d}^{2} \mathbf{r}[\mathbf{n} \times((\nabla \otimes \mathbf{b}) \cdot \mathbf{a})]=\underbrace{\oint_{S} \mathrm{~d}^{2} \mathbf{r}[\mathbf{n} \times \nabla(\mathbf{a} \cdot \mathbf{b})]}_{\overline{\mathbf{0}}}-\oint_{S} \mathrm{~d}^{2} \mathbf{r}[\mathbf{n} \times((\nabla \otimes \mathbf{a}) \cdot \mathbf{b})] \\
=\oint_{S} \mathrm{~d}^{2} \mathbf{r}\left[\nabla_{\mathbf{a}} \times((\mathbf{n} \otimes \mathbf{b}) \cdot \mathbf{a})\right],
\end{aligned}
$$

where $\mathbf{a}(\mathbf{r})$ and $\mathbf{b}(\mathbf{r})$ are arbitrary smooth on $S$ vector fields, while the zeroing out of the term in the middle again follows from the Stokes theorem (Eq. (A1.43) of Ref. [9]) and the absence of a boundary for a closed surface. Note that the right-hand side of Eq. (45) exactly cancels the curl of the last term in Eq. (38). This is expected since the remaining volume integral in Eq. (44) does not depend on the shape of $S_{\delta}$, owing to the weak $\left(R^{-2}\right)$ singularity of the integrand [cf. Eq. (19)]. ${ }^{5}$

Adding another curl and using Eqs. (43)-(45), we get

$$
\begin{gathered}
\nabla \times \nabla \times \mathbf{E}(\mathbf{r})-k_{1}^{2} \mathbf{E}(\mathbf{r})=\lim _{\delta \rightarrow 0} \int_{V_{\mathrm{int}} \backslash V_{\delta}} \mathrm{d}^{3} \mathbf{r}^{\prime}\left[\nabla \times \nabla \times \overline{\mathbf{G}}\left(\mathbf{r}, \mathbf{r}^{\prime}\right)-k_{1}^{2} \overline{\mathbf{G}}\left(\mathbf{r}, \mathbf{r}^{\prime}\right)\right] \cdot \mathbf{j}\left(\mathbf{r}^{\prime}\right) \\
-\lim _{\delta \rightarrow 0} \oint_{S_{\delta}} \mathrm{d}^{2} \mathbf{r}^{\prime}\left[\mathbf{n} \times\left(\nabla \times \overline{\mathbf{G}}\left(\mathbf{r}, \mathbf{r}^{\prime}\right)\right)\right] \cdot \mathbf{j}\left(\mathbf{r}^{\prime}\right)+\overline{\mathbf{L}} \cdot \mathbf{j}(\mathbf{r})=(\overline{\mathbf{I}}-\overline{\mathbf{L}}) \cdot \mathbf{j}(\mathbf{r})+\overline{\mathbf{L}} \cdot \mathbf{j}(\mathbf{r})=\mathbf{j}(\mathbf{r}),
\end{gathered}
$$

where the volume integral vanishes owing to Eq. (14) and the surface integral is evaluated analogously to Eq. (25), but with $\mathbf{E}_{\text {sca }}\left(\mathbf{r}^{\prime}\right)$ multiplied from the left replaced by $\mathbf{j}(\mathbf{r})$ multiplied from the right and with an extra minus sign due to the change of the integration variable.

An alternative option is to start with Eq. (39), leading to

$$
\nabla \times \nabla \times \mathbf{E}(\mathbf{r})-k_{1}^{2} \mathbf{E}(\mathbf{r})=-\left(\nabla^{2}+k_{1}^{2}\right) \int_{V_{\mathrm{int}}} \mathrm{d}^{3} \mathbf{r}^{\prime} g\left(\mathbf{r}, \mathbf{r}^{\prime}\right) \mathbf{j}\left(\mathbf{r}^{\prime}\right)=\mathbf{j}(\mathbf{r}),
$$

where we have used Eq. (13) and

$$
\nabla \times \nabla \times \mathbf{a}-(\nabla \otimes \nabla) \cdot \mathbf{a}=\nabla \times \nabla \times \mathbf{a}-\nabla(\nabla \cdot \mathbf{a})=-\nabla^{2} \mathbf{a}
$$

for an arbitrary smooth $\mathbf{a}(\mathbf{r})$. At the first sight, the differentiation and integration cannot be interchanged in Eq. (48), since the resulting singularity will be of order $R^{-3}$ and thus nonintegrable. However, Appendix B of Ref. [20] shows that the interchange can be done, albeit without an explicit treatment of some intermediate surface integrals. This reference also explains why a direct proof is important, and why many previous derivations do not fully demonstrate the equivalence of the differential and integral Maxwell equations.

To derive the boundary conditions (7), we first note that the limit of $\delta \rightarrow 0$ in Eq. (37) can be replaced by the requirement of a small enough exclusion volume $V_{0}$ (not necessarily connected). In particular,

$$
\mathbf{E}_{\mathrm{sca}}(\mathbf{r})=\int_{V_{\mathrm{int}} \backslash V_{0}} \mathrm{~d}^{3} \mathbf{r}^{\prime} \overline{\mathbf{G}}\left(\mathbf{r}, \mathbf{r}^{\prime}\right) \cdot \mathbf{j}\left(\mathbf{r}^{\prime}\right)+\int_{V_{0}} \mathrm{~d}^{3} \mathbf{r}^{\prime}\left[\overline{\mathbf{G}}\left(\mathbf{r}, \mathbf{r}^{\prime}\right)-\overline{\mathbf{G}}_{\mathrm{st}}\left(\mathbf{r}, \mathbf{r}^{\prime}\right)\right] \cdot \mathbf{j}\left(\mathbf{r}^{\prime}\right)-\frac{\overline{\mathbf{L}}\left(\partial V_{0}, \mathbf{r}\right) \cdot \mathbf{j}(\mathbf{r})}{k_{1}^{2}},(50)
$$

\footnotetext{
${ }^{5}$ Note also that the $R^{-2}$ term is linear in $\mathbf{R}$ and hence vanishes when integrated over a small spherically symmetric volume.
} 
where

$$
\overline{\mathbf{L}}(S, \mathbf{r}) \stackrel{\text { def }}{=} \oint_{S} \mathrm{~d}^{2} \mathbf{r}^{\prime} \frac{\mathbf{n} \otimes \mathbf{R}^{\prime}}{4 \pi R^{3}}
$$

is a generalization of Eq. (27), and we have implicitly used

$$
\int_{V} \mathrm{~d}^{3} \mathbf{r}^{\prime} \overline{\mathbf{G}}_{\mathrm{st}}\left(\mathbf{r}, \mathbf{r}^{\prime}\right)=-\overline{\mathbf{L}}(\partial V, \mathbf{r})
$$

as a consequence of the dyadic Stokes theorem. The integral over $V_{0}$ in Eq. (50) is regular, owing to Eq. (17) and the smoothness of $\mathbf{j}(\mathbf{r})$ (up to the boundary). It is related to the finite-size correction of the dipole polarizability (the so-called $\overline{\mathbf{M}}$ term) in the DDA [5], and its magnitude is $\mathcal{O}\left(d^{2}\|\mathbf{j}(\mathbf{r})\|\right)$, where $d$ is the largest dimension (diameter) of $V_{0}$.

Second, we consider $\mathbf{r}$ to be already very close to the interface between the domains $V_{1}$ and $V_{2}$ (one of them can be $V_{\text {ext }}$ ), so that the boundary is locally flat. Then, we choose $V_{0}$ to consist of two strongly oblate rectangular prisms on both sides of the interface with dimensions $h \times d \times d$ ( $h \ll$ d) and the smallest dimension oriented along the normal $\mathbf{n}$ to the interface (see Fig. 3). We also assume that $d$ is much smaller than both the wavelength in the outer medium $2 \pi / \mathfrak{R}\left(k_{1}\right)$ and the interface curvature. Next, consider the variation of $\mathbf{r}$ over the central line of the prisms along $\mathbf{n}$ at a distance not larger than $h / 2$ from the interface (on either side of the interface, but not exactly on it). The first volume integral in Eq. (50) is smooth over the whole line and has no jump discontinuities over the interface, owing to $\left\|\mathbf{r}-\mathbf{r}^{\prime}\right\| \geq h / 2$. The second integral is negligibly small and also smooth over $\mathbf{r}$. The remaining $\overline{\mathbf{L}}$ term may have discontinuities due to either $\overline{\mathbf{L}}\left(\partial V_{0}, \mathbf{r}\right)$ or $\mathbf{j}(\mathbf{r})$. However, $\overline{\mathbf{L}}$ is the sum of those for each prism; while for a strongly oblate prism it equals $\mathbf{n} \otimes \mathbf{n}$ and $\overline{\mathbf{0}}$ for an arbitrary $\mathbf{r}$ inside and outside the prism, respectively [11]. Thus, $\overline{\mathbf{L}}\left(\partial V_{0}, \mathbf{r}\right)=\mathbf{n} \otimes \mathbf{n}$ over the whole line, ${ }^{6}$ and we finally obtain

$$
\mathbf{E}_{1}(\mathbf{r})-\mathbf{E}_{2}(\mathbf{r})=\mathbf{n}\left[\mathbf{n} \cdot\left(\mathbf{j}_{2}(\mathbf{r})-\mathbf{j}_{1}(\mathbf{r})\right)\right], \quad \mathbf{r} \in S_{\text {int }},
$$

where we have used the continuity of $\mathbf{E}_{\text {inc }}(\mathbf{r})$ across the interface. Equation (53) implies the continuity of both $\mathbf{n} \times \mathbf{E}(\mathbf{r})$ and $\mathbf{n} \cdot \varepsilon(\mathbf{r}) \mathbf{E}(\mathbf{r})$ across the interface. The above derivation is yet another example of the advantage of using an arbitrary (not necessarily spherical) exclusion volume.

\footnotetext{
${ }^{6}$ One can also start with a shape other than the prism (e.g., a hemisphere), as long as it contains a flat part of the boundary. Then as $\mathbf{r}$ approaches this flat part from inside, $\overline{\mathbf{L}}$ would approach the same limit.
} 


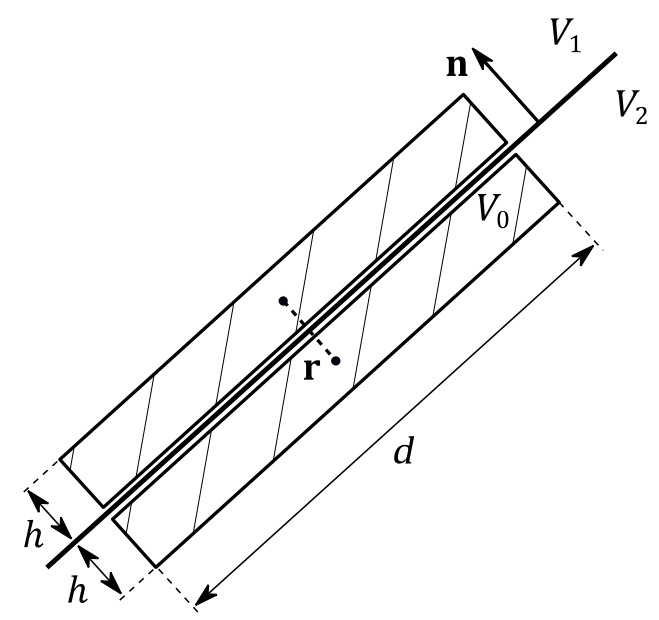

Fig. 3. An exclusion domain for deriving the boundary condition for $\mathbf{E}(\mathbf{r})$ consisting of two oblate rectangular prisms. The width perpendicular to the image (not shown) also equals $d$. Gaps between the prisms and the locally-flat interface are shown solely for convenience. The dashed central line denotes the variation of $\mathbf{r}$ used to calculate the limit when approaching the interface.

The continuity of $\mathbf{H}(\mathbf{r})$, or, equivalently, of $\nabla \times \mathbf{E}_{\mathrm{sca}}(\mathbf{r})$, across the interface follows from Eqs. (37), (44), and (45) and the regularity of the remaining volume integral as discussed above. The radiation condition (9) immediately follows from Eqs. (37) and (16) as a consequence of the regularity of the integrals for $\mathbf{r} \in V_{\text {ext }}$, as discussed in the case of Eq. (42).

To conclude this section we note that the above derivations can in principle be made shorter using the calculus of distributions (generalized functions); see, e.g., Ref. [9]. But that would not necessarily be clearer, since then the singularities of the integrals would be handled implicitly. Moreover, the above derivations apply automatically to the case of interfaces with a finite number of vertices, edges, and even intersections, except for points $\mathbf{r}$ approaching a surface singular point (since then the fields are not necessarily bounded, but integrable). The boundary conditions (7) are then valid at all points on the surface except for the singular ones.

\section{EXISTENCE AND UNIQUENESS OF SOLUTION}

A detailed discussion of existence and uniqueness requires mathematical concepts that are beyond the scope of this paper. Thus we mostly consider and generalize the previously published results, which are somewhat fragmentary since each publication imposes different limitations on the constitutive parameters of the scatterer. And we switch to using $\varepsilon$ (instead of $m$ ) to simplify the discussion of those limitations. Also note that owing to the equivalence shown in Section VI, we discuss the scattering problem in general (Section II), that is, not limited to a particular integral equation (38). 
The analysis of the VIE is the easiest when $\varepsilon(\mathbf{r})$ is smooth in the whole $\mathbb{R}^{3}$, i.e., there is no sharp boundary $[15,21]$; then the classical Riesz-Fredholm theory of integral operators can be applied to prove both existence and uniqueness of solution. In Ref. [21], a positive $\mathfrak{R}(\varepsilon(\mathbf{r}))$ is assumed from the outset (in addition to the assumption $\mathfrak{J}(\varepsilon(\mathbf{r})) \geq 0$ ), while Ref. [15] mentions without proof that a sufficient condition of uniqueness is $\mathfrak{J}\left(\varepsilon_{2}(\mathbf{r})\right)>0$ for any passive host medium. ${ }^{7}$ The latter condition is physically reasonable, since any nontrivial solution of the homogeneous equation (38) (i.e., without $\mathbf{E}_{\text {inc }}$ ) would lose energy inside the particle.

For particles with smooth (potentially nested) surfaces, both the existence and the uniqueness of solution have also been explicitly demonstrated [12,14], although those papers analyze the integro-differential equation (39). Moreover, Ref. [14] considers only real positive $k_{1}$ and $\varepsilon(\mathbf{r})$, while Ref. [12] considers an arbitrary passive host medium $\left(\mathfrak{R}\left(k_{1}\right)>0\right.$ and $\left.\mathfrak{I}\left(k_{1}\right) \geq 0\right)$ and $\mathfrak{R}(\varepsilon(\mathbf{r}))>c_{0}$ (an arbitrary positive constant) ${ }^{8}$ and $\mathfrak{I}(\varepsilon(\mathbf{r})) \geq 0$. If the latter inequality is strict almost everywhere in $V_{\text {int }}$ then $\varepsilon(\mathbf{r})$ does not need to be smooth. Similar conditions of smooth $\varepsilon(\mathbf{r})$ with a strictly positive real part are used in Chapter VI of Ref. [2]. Chapter 3.5 of Ref. [3] proves that the scattering problem has a unique solution for $\mathfrak{J}(\varepsilon(\mathbf{r}))>c_{0}$ almost everywhere in $V_{\text {int }}$ (with no limitations on $\mathfrak{R}(\varepsilon(\mathbf{r}))$ ), but considers only a non-absorbing host medium. Alternatively, a simple proof of uniqueness has been given in Chapter 9.1 of Ref. [16] using the Rellich lemma, but it applies only to a positive real $\varepsilon(\mathbf{r})$ and any passive host medium.

It is important to note that all of the above conditions on constitutive parameters are sufficient, but not necessary for both uniqueness and existence. That is why there is no contradiction in the differences between those conditions. The necessary conditions are intimately related to the spectrum of the linear integral operator $\mathcal{A}$ defined by Eq. (38):

$$
\mathcal{A E}=\mathcal{E}_{\text {inc }} .
$$

Recall that the operator spectrum is a set of complex numbers $\lambda$ such that $(\mathcal{A}-\lambda \mathcal{J})$ does not have a bounded inverse, where $\mathcal{J}$ is the identity operator. This spectrum consists of a discrete spectrum (isolated eigenvalues) ${ }^{9}$ for which there exists a bounded solution of $\mathcal{A} \mathcal{E}=\lambda \mathcal{E}$, and an essential spectrum for which $(\mathcal{A}-\lambda \mathcal{J})^{-1}$ exists but is unbounded [15,22]. The lack of uniqueness and existence of the scattering problem is, then, equivalent to 0 belonging to the discrete and essential spectrum of $\mathcal{A}$, respectively.

For an everywhere smooth $\varepsilon(\mathbf{r})$ (and a passive host medium), the essential spectrum exactly corresponds to the image (set of all values) of the function $\varepsilon\left(\mathbb{R}^{3}\right)$ [15], independently of the size or

\footnotetext{
${ }^{7}$ Note that here the absolute permittivity is used instead of the relative one.

${ }^{8}$ This condition is used to prove the coerciveness of a certain sesquilinear form.

${ }^{9}$ This may also contain a continuous part, but we do not discuss it further.
} 
shape of the scatterer. For homogeneous scatterers with sharp boundaries, there are certain indications that it spans a line from 1 to $\varepsilon$ [15,23], as if there is a narrow smooth transition at the boundary. This is equivalent to Eq. (38) being not solvable for a negative real $\varepsilon$. Recently, Costabel et al. [24] proved (by considering only a non-absorbing host medium) that the essential spectrum consists only of points $1, \varepsilon$, and $(\varepsilon+1) / 2$. Markkanen [22] generalized this result to a particle with edges and vertices, adding intermediate values determined by the corresponding solid angles. However, the essential spectrum is not invariant to the point-wise multiplication [15], e.g., by $\varepsilon(\mathbf{r})-1$, which is used to transition between several equivalent forms of the VIE, using the electric field, induced current, or potential as the main variable [25]. Moreover, the remainder of the line from 1 to $\varepsilon$ contains eigenvalues corresponding to static $\left(k_{1} \rightarrow 0\right)$ shape resonances, which follows from physical reasoning that a positive real $\varepsilon$ cannot support resonances [23]. For instance, a sphere much smaller than the wavelength has a resonance for $\varepsilon=-2$, thus the corresponding $\mathcal{A}$ has an eigenvalue for $(\varepsilon+2) / 3$ [22]. More generally, Budko et al. [26] proved that the eigenvalues of the static scattering operator are contained in the convex hull of $\varepsilon\left(\mathbb{R}^{3}\right)$, denoted hereinafter as $\operatorname{Conv}\left(\varepsilon\left(\mathbb{R}^{3}\right)\right) .{ }^{10}$ To conclude, a practical solution of the VIE for a non-positive real $\varepsilon$ is at least problematic, so one may prefer to avoid this region as a necessary condition for a well-behaved solution.

Additional eigenvalues of $\mathcal{A}$ appear with increasing $\left|k_{1}\right|$. We are not aware of any general bounds on these "resonant" eigenvalues for an arbitrary $\varepsilon(\mathbf{r})$, apart from the uniqueness conditions discussed at the beginning of this section. Thus, we limit the discussion to a homogeneous scatterer with a relative permittivity $\varepsilon$. Then the integral operator $\mathcal{A}$ can be decomposed as follows [cf. Eq. (38)]:

$$
\mathcal{A}=\mathcal{J}+(\varepsilon-1) \mathcal{G}\left(k_{1}\right),
$$

where $\mathcal{G}$ depends on the scatterer geometry and $k_{1}$, but not on $\varepsilon$. In particular, the spectrum of $\mathcal{G}(0)$ belongs to the interval $[0,1]$, as discussed above. Discrete eigenvalues for such a scatterer are directly related to so-called morphology-dependent resonances, which are mostly studied in the framework of the Lorenz-Mie theory for a single sphere [27]. The latter reduces to finding the poles of the Mie coefficients $a_{n}$ and $b_{n}$, or, equivalently, the zeroes of their denominators; this can be summarized as

$$
\exists l \in \mathbb{N}: \eta_{l}(m, x)=0,
$$

where $x=k_{1} a$ is the complex size parameter ( $a>0$ is the fixed sphere radius) and $l$ numbers both the order and type of the Mie coefficients. Each function $\eta_{l}$ can be expressed in spherical Bessel

\footnotetext{
${ }^{10}$ The original proof is for an everywhere smooth $\varepsilon(\mathbf{r})$, but it can be extended to a piecewise smooth one.
} 
and Hankel functions; importantly, it is an analytic function of two complex variables. Any solution $(m, x)$ of Eq. (56) is equivalent to $-1 /\left(m^{2}-1\right)$ belonging to the spectrum of $\mathcal{G}(x / a)$.

Many simulations have shown that resonant values of $m$ are in the fourth quadrant for $x>0$ (real positive $x$ ) $[23,28,29],{ }^{11}$ which naturally corresponds to the condition $\mathfrak{I}(\varepsilon)<0$. In this case the eigenvalues of $\mathcal{G}\left(k_{1}\right)$ have a negative imaginary part, which has been confirmed by direct calculations for the discretized operator [23]. Alternatively, one may consider a fixed $m$ and search for zeroes of $\eta_{l}$ as a function of $x$. For $m>0$, they are located in the fourth quadrant of the complex plane, which corresponds to $\mathfrak{I}\left(\varepsilon_{1}\right)<0$ [30,31]. Both these special cases imply that $x m$ is in the fourth quadrant (or $\mathfrak{I}\left(\varepsilon_{2}\right)<0$ ) at a pole.

Only several papers consider $x$-poles for complex $m$. Hunter et al. [32] studied the shifting of these poles when a small imaginary part is added to a real $m$, using the Taylor expansions. The calculations were performed for 12 specific poles (resonances), all close to the real positive axis in the complex plane of $x$ (for an initial real $m$ ). Interestingly, one can rewrite both the change of $m$ and shifts of the $x$-poles as rotations (change of the complex arguments); then the corresponding changes of the complex arguments satisfy $\Delta \arg (x) \approx-\Delta \arg (m)$, implying $\arg (m x) \approx$ const. In particular, the value of the ratio $\Delta \arg (x) / \Delta \arg (m)$ is between 0.90 and 0.98 for the resonances considered in Ref. [32]. This value being slightly smaller than 1 is confirmed by formulae for the resonance width given by Eq. (47) of Ref. [33], which is directly related to $\arg (x)$ at the pole. Unfortunately, the above first-order analysis cannot definitively answer whether $\mathfrak{I}(m x)$ and $\mathfrak{I}\left(\varepsilon_{2}\right)$ always stay negative (as they are for a real $m$ ). Videen et al. [34] showed that the second order of the Taylor expansions may be significant for very small values of $\mathfrak{J}(m)$. Moreover, a direct calculation of the trajectory of a single specific pole (Fig. 4a of Ref. [34]) was consistent with $\mathfrak{\Im}\left(\varepsilon_{2}\right)<0$.

Similar results (negative-imaginary-part eigenvalues of $\mathcal{G}\left(k_{1}\right)$ for $k_{1}>0$ ) have been shown for cubes [15,35]. Moreover, a single simulation of a vacuum cube inside an absorbing host medium with $\mathfrak{R}\left(\varepsilon_{1}\right)=1$ led to the spectrum consistent with both $\mathfrak{I}\left(\varepsilon_{2}\right)<0$ and $\mathfrak{I}(\varepsilon)<0$ [15].

To conclude this section, we put forward a conjecture that for any homogeneous scatterer inside a passive host medium the "singular" values of $\varepsilon_{2}$ (corresponding to the essential, static, and dynamic spectra) satisfy either $\varepsilon<0$ or both $\mathfrak{I}\left(\varepsilon_{2}\right)<0$ and $\mathfrak{I}(\varepsilon)<0$. This is equivalent to $\varepsilon_{2}$ belonging to the complex wedge: $\pi+\arg \left(\varepsilon_{1}\right) \leq \arg \left(\varepsilon_{2}\right)<2 \pi$, where we assume the range of arg function to be $[0,2 \pi)$ for convenience. Thus, the sufficient conditions for uniqueness and existence of solution (for arbitrary size and shape) are conjectured to be

\footnotetext{
${ }^{11}$ Some of the cited papers use the $\exp (\mathrm{i} \omega t)$ notation, which corresponds to a reverse sign of the imaginary parts of $m$, $\varepsilon, k$, etc. When discussing their conclusions, we implicitly transform them to conform to the notation used in this paper.
} 


$$
0 \leq \arg \left(\varepsilon_{2}\right)<\pi+\arg \left(\varepsilon_{1}\right),\left|\varepsilon_{2}\right|>0 .
$$

Equation (57) is illustrated in Fig. 4, separately for $\varepsilon_{2}$ and $\varepsilon$, defining the allowed regions $Z_{2}$ and $Z$, respectivly. The negative real $\varepsilon_{2}$ are non-physical for the material properties, but the VIE causes no issues with that if the host medium is absorbing.

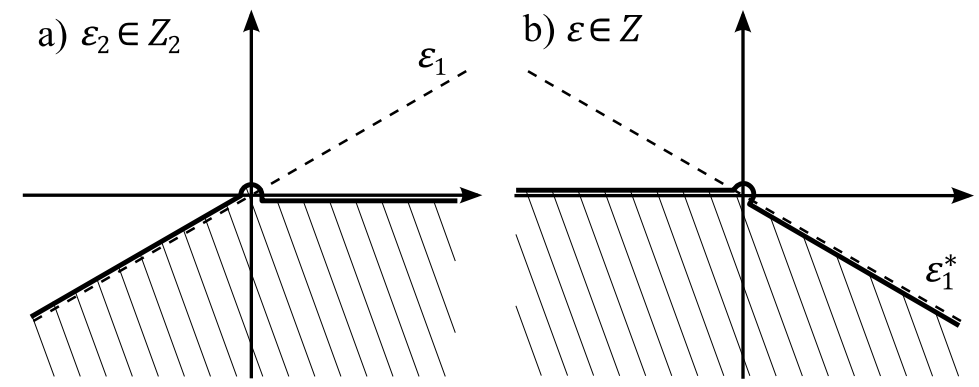

Fig. 4. Conjectured sufficient conditions for the existence and uniqueness of the solution of the scattering problem in a passive host medium, described as blank areas $\left(Z_{2}\right.$ and $\left.Z\right)$ in the complex plane for (a) $\varepsilon_{2}$ and (b) $\varepsilon$, respectively. The shaded areas contain different kinds of resonances (see text). The dashed lines in (a) and (b) extend from the origin through the values of $\varepsilon_{1}$ and its complex conjugate, respectively.

For inhomogeneous scatterers, an additional condition is that the corresponding singular domains be not approached by $\varepsilon$ or $\varepsilon_{2}$ infinitely close, i.e.,

$$
\exists c_{0}>0: \forall \mathbf{r} \in \mathbb{R}^{3}, 0 \leq \arg \left(\varepsilon_{2}(\mathbf{r})\right)<\pi+\arg \left(\varepsilon_{1}\right)-c_{0} \text { and }\left|\varepsilon_{2}(\mathbf{r})\right|>c_{0}
$$

or, equivalently, that the closure ${ }^{12}$ of the set of all values of $\varepsilon_{2}(\mathbf{r})$, denoted $\overline{\varepsilon_{2}\left(V_{\text {Int }}\right)}$, satisfy Eq. (57) pointwise, i.e., $\overline{\varepsilon_{2}\left(V_{\mathrm{Int}}\right)} \subset Z_{2}$. Consistent with the abovementioned results of Budko et al. [26], we also require that $\operatorname{Conv}\left(\overline{\varepsilon_{2}\left(V_{\text {Int }}\right)}\right)$ not contain the origin, which is equivalent to $\varepsilon_{2}\left(V_{\text {int }}\right)$ being contained in the ring sector centered at the origin of the complex plane (a circular sector excluding the neighborhood of the origin) with an opening angle smaller than $\pi$, or to

$$
\exists c_{0}>0: \forall \mathbf{r}, \mathbf{r}^{\prime} \in \mathrm{V}_{\text {int }},\left|\arg \left(\varepsilon_{2}(\mathbf{r})\right)-\arg \left(\varepsilon_{2}\left(\mathbf{r}^{\prime}\right)\right)\right|<\pi-c_{0} .
$$

Moreover, Eqs. (58) and (59) can be combined into the single condition

$$
\operatorname{Conv}\left(\overline{\varepsilon_{2}\left(V_{\text {Int }}\right)}\right) \subset Z_{2} \Leftrightarrow \operatorname{Conv}\left(\overline{\varepsilon\left(\mathbb{R}^{3} \backslash S_{\text {Int }}\right)}\right) \subset Z,
$$

where we have made use of the fact that in the first part $\varepsilon_{2}\left(V_{\text {int }}\right)$ may be augmented by $\varepsilon_{1},{ }^{13}$ so that the equivalence follows from the rotation and scaling of the complex plane. We have also excluded all discontinuities from the image of $\varepsilon$, since the corresponding values $\varepsilon\left(S_{\text {int }}\right)$ are not relevant for the scattering problem and can be arbitrary. This is further discussed in Section IX.

\footnotetext{
12 The set plus all its limiting points (the boundary), further denoted by the overline (not to be confused with the dyadics).

${ }^{13}$ In other words, the condition is equivalent to the same but with $\varepsilon_{2}\left(V_{\text {int }}\right) \cup\left\{\varepsilon_{1}\right\}$, since the line from $\varepsilon_{1}$ to any point in the blank area of Fig. 4(a) is always entirely within this area.
} 
It cannot be overstressed that the above is only a conjecture. While it conforms to all the conclusions of the above-discussed literature, and somewhat generalizes them, we are not aware of any general proof. That should be the topic of future research. As an additional note, when the size and shape of a scatterer are fixed, only a set of discrete points and, possibly, a line in the shaded domain of $\varepsilon$ (Fig. 4(b)) are actually singular. Thus, for most of the points in this domain the scattering problem has a unique solution. However, if the shape and size of the scatterer are arbitrarily varied, these discrete points will move through the whole singular domain and probably cover it entirely. Thus, an additional hypothesis is that Eq. (57) is also a necessary condition if the uniqueness and existence of solution are required for scatterers of all sizes and shapes.

\section{PARTICLES WITH EDGES AND VERTICES}

In this section we further generalize the particle geometry by allowing a finite number of edges and vertices, both as singularities of an otherwise regular surface and as intersections of several regular surfaces. An example of such irregular geometry is shown in Fig. 5, where sharp corners of the 2D image can be interpreted both as point vertices and as sharp edges of the 3D particle shape. Importantly, we still define $S_{i}$ as maximal connected components of $S_{\text {int }}$, keeping Eqs. (5) and (6) valid.

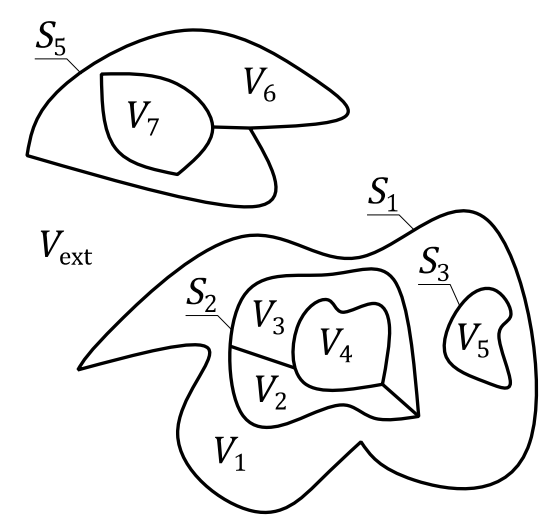

Fig. 5. An example of a multi-body multi-layered scatterer with piece-wise smooth boundaries and interfaces (having a finite number of edges and vertices). Each $S_{i}$ is a closed connected surface, but not necessarily a regular one; it separates at least two domains, one of which may be the external medium.

The boundary conditions (7) are still valid for all parts of $S_{\text {int }}$, except for singularities, but alone they are no longer sufficient to make the problem unambiguous. Additional assumptions must be invoked, e.g., that the charges and currents localized at shape singularities are zero, i.e., they do not radiate any energy (Chapter 9.2 of Ref. [16]): 


$$
\lim _{\delta \rightarrow 0} \oint_{S_{\delta}} \mathrm{d}^{2} \mathbf{r} \mathbf{n} \cdot \mathfrak{R}\left[\mathbf{E}(\mathbf{r}) \times \mathbf{H}(\mathbf{r})^{*}\right]=0,
$$

where the closed surface $S_{\delta}$ contracts around the edge or vertex, e.g., having the shape of a capped curved cylinder and sphere, respectively. This has also been stated to be equivalent to the physically reasonable requirement of locally finite energy of the electromagnetic field (i.e., the energy is finite inside any bounded volume) or that $\mathbf{E}(\mathbf{r})$ and $\mathbf{H}(\mathbf{r})$ are locally square-integrable $[3,16]$, and guarantees finite charge and currents on the whole $S_{\text {int }}$. Unfortunately, we are not aware of a detailed discussion of this equivalence in the literature. Thus, we further consider the locally squareintegrability of the fields as a primary assumption and note that Eq. (61) follows from it and the Poynting theorem [4]:

$$
\oint_{\partial V} \mathrm{~d}^{2} \mathbf{r} \mathbf{n} \cdot\left[\mathbf{E}(\mathbf{r}) \times \mathbf{H}(\mathbf{r})^{*}\right]=-\mathrm{i} \omega \int_{V} \mathrm{~d}^{3} \mathbf{r}\left(\varepsilon(\mathbf{r})^{*}|\mathbf{E}(\mathbf{r})|^{2}-\mu_{0}|\mathbf{H}(\mathbf{r})|^{2}\right) .
$$

Importantly, Eq. (62) can be applied to surfaces $\partial V$ crossing the scatterer interfaces, since Eq. (7) implies the continuity of the integrand over the interfaces ${ }^{14}$ and the integration surface can be deformed to circumvent the interfaces (Fig. 6). The integrability of the integrand on the right-hand side of Eq. (62) implies that it can be considered both over the finite volume enclosing the shape singularity $(V)$ and over the same volume with the singularity excluded $\left(V \backslash V_{\delta}\right)$, and the result is the same in the limit $\delta \rightarrow 0$. But the difference between these two approaches on the left-hand side of Eq. (62) is exactly Eq. (61) before taking the real part. ${ }^{15}$
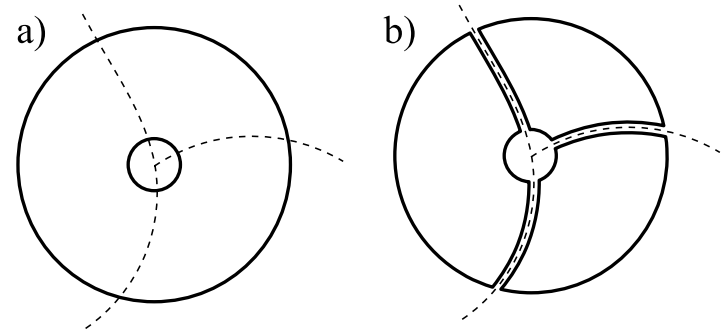

Fig. 6. An example of the deformation of the integration surface to circumvent the scatterer interfaces (shown by dashed curves), from (a) to (b). The surface integral does not change for any integrand that is continuous across the interfaces, while the volume integral is the same for any integrable function.

\footnotetext{
${ }^{14} \mathbf{n} \cdot\left(\mathbf{E} \times \mathbf{H}^{*}\right)=-(\mathbf{n} \times \mathbf{E}) \cdot\left[\mathbf{n} \times\left(\mathbf{n} \times \mathbf{H}^{*}\right)\right]$

${ }^{15}$ Proving the reverse implication (from Eq. (61) to square integrability of the fields) is substantially more involved. The real part of Eq. (62) directly implies only integrability of $\mathfrak{I}(\varepsilon(\mathbf{r}))|\mathbf{E}(\mathbf{r})|^{2}$, which is not helpful if the medium is neither absorbing nor active in the neighborhood of the shape singularity. In this case, one may assume that Eq. (61) is valid before taking the real part, but that is unjustified unless one relates the regularity of the real and imaginary parts of Eq. (61) using general properties of the electromagnetic field, e.g., through the VIE. We leave the latter for future research.
} 
Stronger conditions have also been proposed, e.g., the "tip condition" that singularities of $\mathbf{E}$ and $\mathbf{H}$ be weaker than $R^{-1}$ has been proven sufficient for an infinite cone [36]. Evidently, the tip condition also implies Eq. (61) and square integrability of the fields. To summarize, the fields can be unbounded when approaching shape singularities, but only weakly so in the sense specified above.

In discussing the VIE in the presence of edges and vertices, we first note that the above condition of locally square-integrable fields is convenient to have anyway, since otherwise the whole application of the VIE (the calculation of the integrals involved) is ambiguous. Although we avoided function spaces from the outset, any mathematically rigorous discussion of the VIE includes a specific function space in which the solution is searched for. In terms of $\mathbf{E}(\mathbf{r})$ alone, it is typically the space in which both the function and its curl are locally square-integrable $[13,14,37]$. Thus, given this physical assumption, the VIE seems to be directly applicable to particles with edges without any changes. In particular, this conclusion is supported by the successful numerical application of the DDA to a cube, exhibiting even better accuracy than that for smooth shapes [6]. However, the above derivations connecting the VIE with the differential Maxwell equations do require some modifications.

First, we denote the $\delta$-neighborhoods of the shape singularities as $V_{1}^{\delta}, V_{2}^{\delta} \ldots$, bounded by closed surfaces $S_{1}^{\delta}, S_{2}^{\delta}, \ldots$, respectively [cf. Eq. (61)]. Some complications arise from the fact that shape singularities may intersect (e.g., edges end up at vertices); in such cases we define the corresponding $S_{i}^{\delta}$ to enclose the whole combined singularity, so that the minimum distance from any point on the surface to the singularity equals $\delta$. For instance, a cube will require only a single such surface, being a union of 8 incomplete spheres and 12 side surfaces of a cylinder. We further define the total volume around the singularities $V_{\mathrm{s}}$ and singularity-excluded domains $V_{i}^{\prime}$ and $V_{\text {ext }}^{\prime}$,

$$
V_{\mathrm{s}} \stackrel{\text { def }}{=} \bigcup_{i} V_{i}^{\delta}, \quad V_{i}^{\prime} \stackrel{\text { def }}{=} V_{i} \backslash V_{\mathrm{s}}, \quad V_{\text {ext }}^{\prime} \stackrel{\text { def }}{=} V_{\text {ext }} \backslash V_{\mathrm{s}}
$$

and partition each interface with excluded singularities $S_{i} \backslash V_{\mathrm{s}}$ into several pair-wise disjoint regular parts $S_{j}^{\mathrm{r}}$ (the latter are numbered sequentially throughout the whole scatterer). Moreover, each $S_{i}^{\delta}$ is partitioned into two or more segments $S_{i, 1}^{\delta}, S_{i, 2}^{\delta}, \ldots$ belonging to different domains (out of $V_{\text {ext }}, V_{1}$, $\left.V_{2}, \ldots\right)$. Any two of these segments touch only on one of $S_{j}^{\mathrm{r}}$ (if at all), which together with Eq. (7) implies that both $\mathbf{n} \times \mathbf{E}(\mathbf{r})$ and $\mathbf{n} \times \mathbf{H}(\mathbf{r})$ are continuous across the whole $S_{i}^{\delta}$. An example of the described singularity exclusion is shown in Fig. 7, illustrating the newly introduced definitions. To avoid ambiguity, we take the default orientation of the normal to $S_{i, j}^{\delta}$ to coincide with the outwardpointing normal to $S_{i}^{\delta}$, while the orientation of the normal to $S_{j}^{r}$ is not relevant for further discussion. 


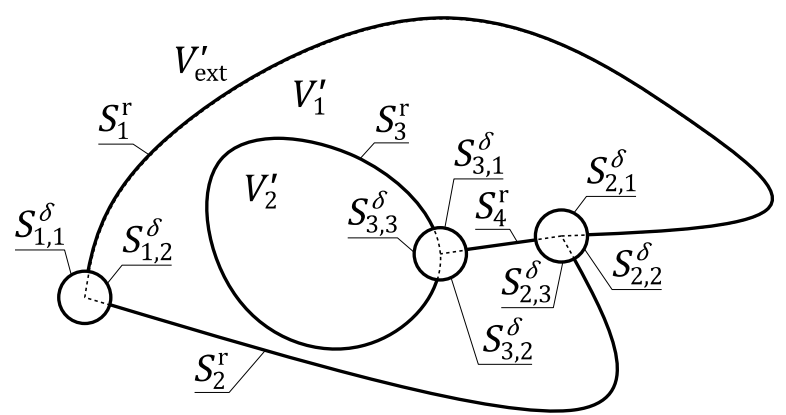

Fig. 7. An example of the exclusion of shape singularities from the interfaces and volume domains. See the main text for an explanation of the symbols; the dashed lines denote the parts of the original irregular surfaces $S_{i}$ (cf. Fig. 5) falling inside the neighborhood of singularities. Each $S_{j}^{\mathrm{r}}$ is a regular connected surface separating exactly two domains, one of which may be the external medium.

To derive the VIE from the differential Maxwell equations, we proceed analogously to Section V. We write down Eq. (34) for each $V_{i}^{\prime}$ and add them up together with Eq. (36) for $V_{\text {ext }}^{\prime} \cdot{ }^{16}$ Each $\partial V_{i}^{\prime}$ is a union of one or more $S_{j}^{\mathrm{r}}$ and zero or more $S_{j, l}^{\delta}$, while $\partial V_{\mathrm{ext}}^{\prime}$ additionally includes $S_{\Delta}$ (in the limit $\Delta \rightarrow \infty)$. In the final sum, each $S_{j}^{\mathrm{r}}$ occurs exactly twice and with opposite signs canceling each other, while each $S_{i, j}^{\delta}$ occurs only once. Applying additionally Eq. (31) and

$$
\chi_{V_{\mathrm{ext}}^{\prime}}(\mathbf{r})+\sum_{j} \chi_{V_{j}^{\prime}}(\mathbf{r})=1-\chi_{S_{\mathrm{int}} \mathrm{U} \bar{V}_{\mathrm{s}}}(\mathbf{r})
$$

[cf. Eq. (41)], where $\bar{V}_{\mathrm{s}} \stackrel{\text { def }}{=} V_{\mathrm{s}} \cup \partial V_{\mathrm{s}}$, we obtain

$$
\mathbf{E}_{\mathrm{sca}}(\mathbf{r})=\lim _{\delta \rightarrow 0} \int_{V_{\mathrm{int}} \backslash\left(V_{\delta} \cup V_{\mathrm{s}}\right)} \mathrm{d}^{3} \mathbf{r}^{\prime} \overline{\mathbf{G}}\left(\mathbf{r}, \mathbf{r}^{\prime}\right) \cdot \mathbf{j}\left(\mathbf{r}^{\prime}\right)-\frac{\overline{\mathbf{L}} \cdot \mathbf{j}(\mathbf{r})}{k_{1}^{2}}+\sum_{i} \oint_{S_{i}^{\delta}} \mathrm{d}^{2} \mathbf{r}^{\prime} \mathbf{X}\left(\mathbf{r}^{\prime}, \mathbf{r}\right)
$$

for $\mathbf{r} \in \mathbb{R}^{3} \backslash\left(S_{\text {int }} \cup \bar{V}_{\mathrm{s}}\right)$.

When we take the limit of $V_{\mathrm{s}}$ contracting to the shape singularities, the volume integral in Eq. (65) behaves regularly owing to $\mathbf{E}(\mathbf{r})$, and hence $\mathbf{j}(\mathbf{r})$, being square-integrable inside $V_{\text {int }}$. Therefore, the limiting result is the integral over $V_{\text {int }} \backslash V_{\delta}$ exactly as in Eq. (37). The only remaining proposition to prove is that

$$
\lim _{\delta \rightarrow 0} \oint_{S_{i}^{\delta}} \mathrm{d}^{2} \mathbf{r}^{\prime} \mathbf{X}\left(\mathbf{r}^{\prime}, \mathbf{r}\right)=0,
$$

where $\delta$ should not be mistaken for the parameter of the volume integral in Eq. (65). For a fixed $\mathbf{r}$, Eq. (66) follows from the trivial analysis of singularity orders. In particular, square integrability of $\mathbf{E}$ and $\mathbf{H}$ inside $V_{\mathrm{s}}$ implies that

\footnotetext{
${ }^{16}$ Strictly speaking, the entire boundaries $\partial V_{i}^{\prime}$ and $\partial V_{\text {ext }}^{\prime}$ must be smooth, which does not hold at junctions between $S_{j}^{\mathrm{r}}$ and $S_{j, l}^{\delta}$. However, the corresponding junctions can be locally smoothed (at a scale much smaller than $\delta$ ) without changing any relevant integrals due to the boundedness of the fields in a small neighborhood of a junction.
} 


$$
|\mathbf{E}|,|\mathbf{H}|=\left\{\begin{aligned}
\sigma\left(\delta^{-1}\right), & \text { near edge, } \\
\sigma\left(\delta^{-3 / 2}\right), & \text { near vertex, }
\end{aligned}\right.
$$

where $\delta$ denotes the distance from the edge or vertex, respectively. Note that Eq. (67) is a weaker condition than the abovementioned tip condition; still it implies

$$
\left|\oint_{S_{i}^{\delta}} \mathrm{d}^{2} \mathbf{r}^{\prime} \mathbf{X}\left(\mathbf{r}^{\prime}, \mathbf{r}\right)\right|=\left\{\begin{array}{rc}
\sigma(1), & \text { near edge, } \\
\sigma\left(\delta^{1 / 2}\right), & \text { near vertex },
\end{array}\right\} \underset{\delta \rightarrow 0}{\longrightarrow} 0 .
$$

For a combined shape singularity with edges and vertices, Eq. (68) is valid for each simple part of $S_{i}^{\delta}$, while there is only finite number of such parts.

To finalize the equivalence we note that the reverse path from the VIE (with a locally-squareintegrable solution) to the differential Maxwell equations and boundary conditions remains exactly the same as in Section VI.

The published literature on the existence and uniqueness of the solution for particles with irregular boundaries is scarce and was partly mentioned in Section VII. In particular, Chapter 9.2 of Ref. [16] proves uniqueness for a positive real $\varepsilon(\mathbf{r})$ and any passive host medium, while Chapter 3.5 of Ref. [3] proves the uniqueness for a non-absorbing host medium and $\mathfrak{I}(\varepsilon(\mathbf{r}))>c_{0}$ almost everywhere in $V_{\text {int }}$. van Beurden and van Eijndhoven [13] also considered a non-absorbing host medium and assumed both $\Re(\varepsilon(\mathbf{r}))$ and $\mathfrak{I}(\varepsilon(\mathbf{r}))$ to be nonnegative and at least one of them positive almost everywhere in $V_{\text {int }} \cdot{ }^{17}$ Thus, the entire Section VII and its concluding conjecture [Fig. 4 and Eq. (60)] remain plausible for general scatterers with irregular boundaries.

To conclude this section, let us reiterate that the VIE is directly applicable to particles with edges and vertices without any modification, and thus can be thought of as being superior to the differential formulation which requires extra assumptions. However, this is not a fundamental difference between the integral and differential formulations, but rather a consequence of a specific problem in which a natural assumption of local square integrability of the VIE solution (a choice of the solution space) is sufficient to eliminate the spurious solutions of the original differential problem. Moreover, not every possible VIE for electromagnetic scattering has this desirable property. It holds if Eq. (38) or (39) is reformulated in terms of $\mathbf{j}(\mathbf{r})$ or $\mathbf{D}(\mathbf{r}) \stackrel{\text { def }}{=} \varepsilon(\mathbf{r}) \mathbf{E}(\mathbf{r})$, requiring only that $\varepsilon(\mathbf{r})$ be nonzero almost everywhere [13]. However, it is not so for the so-called potential VIE (with scalar and vector potentials as unknowns). In particular, when applied to a homogeneous cube with $\varepsilon$ close to certain negative real values, it leads to spurious solutions localized at edges and vertices [22]. Those solutions seem to have nonzero charges/currents on edges and vertices that should be avoided according to Eq. (61). This can be explained by the fact that square integrability

\footnotetext{
${ }^{17}$ There seems to be a minor error in their derivation: the absolute value in Eq. (16) of Ref. [13] should be replaced by the real part for coerciveness to hold. This implies that $\varepsilon(\mathbf{r})$ should not be purely imaginary as well. However, this is not essential, since this case is covered by Cessenat [3] anyway.
} 
of the potentials (naturally occurring for the discretized solution of the integral equation) does not imply square integrability of the fields (due to the extra differentiation). Hopefully, this can be alleviated by a more careful choice of the testing functions for the discretization of the integral operator, as mentioned in Ref. [22].

\section{CONTINUITY WITH RESPECT TO $m(r)$}

All previous sections vividly demonstrate how complexities of the scatterer morphology result in complications of the differential scattering problem (extra assumptions) and derivations of its equivalence to the VIE. In the following, we draft an alternative approach which mostly deals with the simplest case of an everywhere smooth $m(\mathbf{r})$, more specifically, a Hölder-continuous one. In this case the VIE is equivalent to the differential Maxwell equations (without boundary or any additional conditions) - see the discussion following Eq. (38). The corresponding operator is wellbehaved [15,21], as discussed in Section VII, and the solution is smooth. So the main idea is to replace the solution for an arbitrary scatterer (with sharp and irregular interfaces) by the limit of solutions for a smooth $m(\mathbf{r})$.

This idea has been mentioned in various forms in the literature. For instance, Chapter 9.1 of Ref. [16] mentions that the result for an edge can be defined as a limit of the results for a smooth boundary, when the latter is transformed into an edge. Kline [38] proposed a general way to generalize the differential Maxwell equations to encompass discontinuous fields and/or material properties, based on the postulation that the integral representation (not necessarily a VIE) derived for the smooth case directly applies to the discontinuous case. Moreover, it is postulated that the limit of solutions for the continuous case is the proper solution for the discontinuous case, provided the latter allows several solutions. The boundary conditions (7) naturally appear in this approach as an implication of the VIE (see Section VI), which has been mentioned specifically in Ref. [14] as a consequence of assuming that the Maxwell equations are satisfied in $\mathbb{R}^{3}$ in the generalized-function sense. However, we are not aware of any detailed description of this approach, not to mention a rigorous proof. Thus, we start filling this gap in the following, although we may pose more questions than we are currently able to answer.

First, a wide class of discontinuous functions $m(\mathbf{r})$ can be approximated by a sequence of everywhere smooth (Hölder-continuous) $\left\{m_{n}(\mathbf{r})\right\}$ in some functional, e.g., $L^{2}$, norm, i.e.,

$$
\lim _{n \rightarrow \infty} m_{n}=m \text {, }
$$

where handwritten symbols denote functions in contrast to their values at a particular point [cf. Eq. (54)]. We do not give rigorous definitions here, but that is related to the space of smooth functions being dense inside $L^{2}\left(\mathbb{R}^{3} \backslash V_{\text {ext }}\right)$ or a similar space - a standard topic of functional 
analysis. At a minimum, all scatterers with a finite number of irregular interfaces discussed above can be represented in this way.

Second, we note that the operator $\mathcal{A}$ in Eq. (54) implicitly depends on $m$, hence the solution of this equation is given by

$$
\mathcal{E}=\mathcal{A}^{-1}(m) \varepsilon_{\text {inc }} .
$$

The most important part of the whole derivation is the dependence of $\mathcal{E}$ on $m$ for a fixed $\mathcal{E}_{\text {inc }}$, in particular, whether this dependence can be assumed continuous. The conjecture is that

$$
\lim _{n \rightarrow \infty} \mathcal{A}^{-1}\left(m_{n}\right) \mathcal{E}_{\text {inc }}=\mathcal{A}^{-1}(m) \mathcal{E}_{\text {inc }}
$$

in some domain of $m$, where we additionally assume that $\mathcal{A}^{-1}$ is well-defined for $m$ and each of $m_{n}$, i.e., the solution of each respective scattering problem exists and is unique (as discussed below). While the continuity seems reasonable for a smooth resulting $m$, it is not at all evident for discontinuous ones which represent our main interest. The potential failure of Eq. (71) may be caused by several factors: the limit may (i) not exist, (ii) be unbounded (i.e., each $\varepsilon_{n} \stackrel{\text { def }}{=}$ $\mathcal{A}^{-1}\left(m_{n}\right) \mathcal{E}_{\text {inc }}$ is locally square integrable, but its limit is not), or (iii) be not equal to the right-hand side.

The detailed rigorous analysis of the continuity conjecture in proper functional (Sobolev) space remains the subject of future research. On one hand, it is further complicated by the fact that the dependence of $\mathcal{A}$ on $m$ is of the form "identity + linear" [cf. Eq. (55)], which makes $\mathcal{A}^{-1}$ nonlinear with respect to $m$. On the other hand, the nature of this dependence is multiplicative [cf. Eq. (3)], thus making it easily invertible. Moreover, the VIE is probably less sensitive to shape features than surface-integral formulations (see, e.g., Chapter 5 of Ref. [39]). However, it is for the latter that certain continuity has actually been proven, albeit only for perfect conductors with smooth surfaces (see Chapter 7.2 of Ref. [21]).

Third, if $\lim _{n \rightarrow \infty} \varepsilon_{n}$ exists, it is natural to postulate it as the definition for the solution of the scattering problem for an irregular $m$. If, additionally, this limit equals $\mathcal{E}$ from Eq. (70) (i.e., the continuity conjecture holds) then this solution can be obtained from the VIE with no modifications.

While this concludes the template of a proof, we further discuss three related issues which give some additional confidence in the continuity conjecture.

We start with a discussion of the existence and uniqueness, i.e., whether $\mathcal{A}^{-1}(m)$ exists and is bounded and whether it follows from the regularity of $\mathcal{A}^{-1}\left(m_{n}\right)$. Most of the results from Section VII apply but require minor modifications to account for taking a limit. To this end, we analyze the general, albeit hypothetical condition (60). On one hand, wherever $m$ is smooth, the $L^{2}$ convergence of Eq. (69) implies a point-wise convergence, at least for a subsequence of $m_{n}(\mathbf{r})$; hence, 


$$
m\left(\mathbb{R}^{3} \backslash S_{\text {int }}\right) \subset \overline{\bigcup_{n} m_{n}\left(\mathbb{R}^{3}\right)} .
$$

On the other hand, for any piecewise smooth $m$, as considered in Section VIII, we can construct $m_{n}$ to have only the same values as those of $m$ and "in between". Specifically, we set $m_{n}(\mathbf{r})=$ $m(\mathbf{r})$ for all $\mathbf{r}$, except in a small neighborhood $U_{n}$ of $S_{\text {int }}\left(U_{n} \underset{n \rightarrow \infty}{\longrightarrow} S_{\text {int }}\right)$. Inside this neighborhood, $m_{n}(\mathbf{r})$ changes smoothly from the value of $m(\mathbf{r})$ on one side of the boundary to that on the other. A particular S-shaped function to use is not important, but the corresponding intermediate values of $\varepsilon_{n}(\mathbf{r})=m_{n}^{2}(\mathbf{r})$ should all be on the line in the complex plane between the two values of $\varepsilon .{ }^{18}$ Near the interface intersections (see, e.g., Fig. 5), $m_{n}$ (r) should smoothly connect three or more values of $m(\mathbf{r})$ on different sides of the shape singularity with the corresponding intermediate values of $\varepsilon_{n}(\mathbf{r})$ limited to the convex hull of the corresponding values of $\varepsilon$. Thus, ${ }^{19}$

$$
\varepsilon\left(\mathbb{R}^{3} \backslash U_{n}\right) \subset \varepsilon_{n}\left(\mathbb{R}^{3}\right) \subset \operatorname{Conv}\left(\varepsilon\left(\mathbb{R}^{3} \backslash U_{n}\right)\right) \subset \operatorname{Conv}\left(\varepsilon\left(\mathbb{R}^{3} \backslash S_{\text {int }}\right)\right) .
$$

Equations (72) and (73) imply the "continuity" of the condition (60). If it is satisfied for all $m_{n}$ simultaneously and with limiting points, i.e., $\operatorname{Conv}\left(\overline{\mathrm{U}_{n} \varepsilon_{n}\left(\mathbb{R}^{3}\right)}\right) \subset Z$, then the limiting scattering problem is well-defined. Conversely, for any piecewise smooth $m$ satisfying Eq. (60), one can construct a converging sequence of smooth functions $m_{n}$, for each of which the scattering problem is well-defined. The latter supports the validity of using the VIE for such an $m$.

Let us further consider the convergence of the spectrum of $\mathcal{A}\left(m_{n}\right)$. On one hand, Section VII presents some controversial evidence against such convergence. While considering the simplest case of a homogeneous sphere in a vacuum, different authors suggest that the essential spectrum is either a line from 1 to $\varepsilon$ [15] or only three points: -1 , $\varepsilon$, and $(1+\varepsilon) / 2$ [24]. However, for any smooth approximation $m_{n}$ constructed above, the essential spectrum is a line from 1 to $\varepsilon$ [15]; hence, so is its limit. ${ }^{20}$ This apparent difference remains for the whole spectrum of the operator as well, since the latter differs from the essential spectrum only at a discrete set of points.

On the other hand, all these differences disappear if we take the convex hull of the spectrum. Moreover, according to Ref. [26] for arbitrary piecewise smooth scatterer the convex hull of the essential spectrum $\operatorname{Conv}\left(\varepsilon\left(\mathbb{R}^{3} \backslash S_{\text {int }}\right)\right)$ contains the discrete spectrum in the static limit, and, thus, equals the convex hull of the whole spectrum for the static operator $\mathcal{A}_{\text {st }} \stackrel{\text { def }}{=} \mathcal{A}_{k_{1}=0}$. At the same time, Eq. (73) implies

$$
\operatorname{Conv}\left(\varepsilon_{n}\left(\mathbb{R}^{3}\right)\right)=\operatorname{Conv}\left(\varepsilon\left(\mathbb{R}^{3} \backslash U_{n}\right)\right) \underset{n \rightarrow \infty}{\longrightarrow} \operatorname{Conv}\left(\varepsilon\left(\mathbb{R}^{3} \backslash S_{\text {int }}\right)\right) .
$$

\footnotetext{
${ }^{18} \varepsilon_{n}(\mathbf{r})$ should not be confused with $\varepsilon_{2}(\mathbf{r})$; the former is used only with the subscript $n$.

19 The presented proof can be made rigorous for a piecewise smooth $m$ by explicitly (and tediously) constructing the described smooth junctions. However, we are not certain that this can be done for an arbitrary $m$ satisfying Eq. (69).

${ }^{20}$ The intermediate values on this line are thinned out in the sense that they correspond to $U_{n}$ whose volume shrinks to zero. Hence, the effect of this part of the essential spectrum on the solution of the VIE in the $L^{2}$ space is unclear.
} 
So we have a convergence of the convex hull of the spectrum of $\mathcal{A}_{\text {st }}$. This does not tell us anything about the discrete spectrum of the operator for $k_{1} \neq 0$, but we may expect the continuity to hold for those discrete eigenvalues, since the most problematic part of $\overline{\mathbf{G}}$, and hence of $\mathcal{A}$, is the strongly singular static part $\overline{\mathbf{G}}_{\text {st }}$ which fully manifests itself in $\mathcal{A}_{\text {st }}$. The continuity of the convex hull of the spectrum is much weaker than that implied by Eq. (71), but does show some similarity of its leftand right-hand sides, e.g., in terms of their numerical computation. ${ }^{21}$

Thus, we arrive at the convergence of the discretization schemes for the numerical solution of the VIE. The latter can be the topic of a separate review (see, e.g., Refs. [5,22]); here we only note that discretization effectively replaces the integral operator $\mathcal{A}$ with an operator $\mathcal{A}_{N}$ having a finite rank $N$. It is typically assumed that

$$
\lim _{N \rightarrow \infty} \mathcal{A}_{N}^{-1}(m) \mathcal{E}_{\text {inc }}=\mathcal{A}^{-1}(m) \mathcal{E}_{\text {inc }}
$$

which is a manifestation of so-called numerically-exact solutions [37,40] and is realized in practice for the VIE discretized with proper basis and testing functions. Importantly, any bounded finiterank linear operator is equivalent to the matrix and, thus, is continuous:

$$
\lim _{n \rightarrow \infty} \mathcal{A}_{N}^{-1}\left(m_{n}\right) \mathcal{E}_{\text {inc }}=\mathcal{A}_{N}^{-1}(m) \mathcal{E}_{\text {inc }}
$$

We may even choose a sequence of smooth functions $m_{n}$ such that ${ }^{22}$

$$
\mathcal{A}_{N}\left(m_{n}\right) \equiv \mathcal{A}_{N}(m), \quad n \geq N,
$$

but this is not required for the following.

An important hypothesis is the uniform convergence of the limiting sequence in Eq. (75) for all scatterer functions in the neighborhood of $m$, or at least for the sequence $\left\{m_{n}\right\}$. It implies the possibility to interchange the limits leading, along with Eq. (76), to

$$
\lim _{n \rightarrow \infty} \mathcal{E}_{n}=\lim _{n \rightarrow \infty} \lim _{N \rightarrow \infty} \mathcal{A}_{N}^{-1}\left(m_{n}\right) \mathcal{E}_{\mathrm{inc}}=\lim _{N \rightarrow \infty} \mathcal{A}_{N}^{-1}(m) \mathcal{E}_{\mathrm{inc}}=\mathcal{E},
$$

which is exactly the continuity conjecture (71). It is not clear, however, whether proving the uniform convergence of Eq. (75) is fundamentally easier. Conversely, a proof of Eq. (71) would actually justify the use of the discussed numerical methods for a discontinuous $m$, which may seem questionable since they make no distinction between $m$ and $m_{n}$ (for a large enough $n$ for a fixed $N$ ). The latter has been discussed and practically justified in the DDA simulations of light scattering by a cube [6]. Moreover, Eq. (78) can be generalized to describe the convergence of the spectrum of the discretized operator which has been analyzed numerically for a few examples in the framework of the DDA $[19,41]$.

\footnotetext{
${ }^{21}$ The convergence of an iterative solver is determined by the envelope of the spectrum [23], which is not necessarily the convex hull, but rather a simply connected superset of the spectrum. However, the construction of smooth approximations before Eq. (73) can be modified such that $\varepsilon_{n}\left(\mathbb{R}^{3}\right)$ lies within this envelope.

${ }^{22}$ For instance, in the simplest case of the DDA with a cubical discretization and pointwise testing (the collocation method) only the values of $m(\mathbf{r})$ in the centers of the cubes matter. Any smoothing of $m$ between these nodes does not change the discretized operator, e.g., as discussed before Eq. (73).
} 
To finalize this section, we stress once again that the rigorous proof of the continuity conjecture (71) or, more specifically, the evaluation of specific conditions on the underlying functions that make it valid, remains to be done. However, there exists additional supporting physical reasoning. Since the physical properties of the materials are discontinuous at atomic scales, the macroscopic Maxwell equations (1) are typically derived from the microscopic ones by averaging over some finite size $\delta$ [40]. Hence, Eq. (1) is valid only down to the scale of $\delta$, and any variation of $m$ at a smaller scale should not affect the solution. In other words, when using Eq. (1) we implicitly assume that any such variation of $m$ has negligible effect for large enough scatterers, which is similar to assuming the uniform convergence of Eq. (75). The only other option is to rigorously average the microscopic Maxwell equations near the material discontinuities, considering a realistic placement of atoms, the interaction of electrons, etc., which will be entirely daunting near the intersection of several interfaces. Surely, this physical reasoning is not a substitute for a rigorous mathematical proof, but it helps achieve a certain level of mental comfort.

\section{CONCLUSION}

Consistent with the objectives formulated in the Introduction, we have presented a general derivation of the VIE for a very general type of scatterer in the form of an arbitrary spatially finite group of particles, including those with edges, corners, and intersecting internal interfaces, immersed in a passive host medium. We have thoroughly discussed the existence and uniqueness of the VIE solution related to the spectrum of the corresponding integral operator. Moreover, we have shown that the conjectured continuity of the inverse integral operator with respect to the refractiveindex function leads to an even simpler derivation of the VIE. Whenever possible, we have closely followed previously published derivations and constructed a new derivation and new conjectures to fill the existing gaps. Importantly, we believe that the resulting description is reasonably selfcontained and complete, covering the VIE from all possible conceptual perspectives. As such, our paper could also be considered a review of the current state-of-the-art of this subject.

Yet a lot of work remains to be done. First, in order to make the derivations widely accessible, we have refrained from complete mathematical rigor in certain places, e.g., in terms of specific smoothness requirements for the fields and constitutive parameters. This issue seems to be a rather technical one and should be resolvable along the lines of the referenced rigorous accounts. Second, we formulated two important conjectures: (i) the general condition on the electric permittivity of the scatterer and the host medium to guarantee the existence and uniqueness of solution, and (ii) the continuity of the VIE solution with respect to the refractive-index function. To attain the full predictive power, these conjectures need to be rigorously proved with a specification of the function spaces in which they are satisfied. Third, it is highly desirable to extend this complete analysis to 
anisotropic and magnetic materials. Accounting for material anisotropy is straightforward and mostly amounts to replacing the scalar electric permittivity (or refractive index) by a dyadic one and tracing it appropriately through all the derivations. The consideration of magnetic materials should result in replacing a single VIE with a system of two coupled VIEs, for the electric and magnetic fields, respectively. The derivation of such VIEs can be expected to be lengthier, but not fundamentally more involved. However, the existence and uniqueness conditions will require a separate analysis.

\section{ACKNOWLEDGEMENTS}

This work was supported by the Russian Foundation for Basic Research grant No. 18-01-00502, the NASA Radiation Sciences Program, and the NASA Remote Sensing Theory Program.

\section{REFERENCES}

[1] D. S. Saxon, Lectures on the Scattering of Light (Department of Meteorology, University of California at Los Angeles, Los Angeles, USA, 1955).

[2] C. Müller, Foundations of the Mathematical Theory of Electromagnetic Waves (SpringerVerlag, Berlin, 1969).

[3] M. Cessenat, Mathematical Methods in Electromagnetism: Linear Theory and Applications (World Scientific, Singapore, 1996).

[4] M. I. Mishchenko, Electromagnetic Scattering by Particles and Particle Groups: An Introduction (Cambridge University Press, Cambridge, UK, 2014).

[5] M. A. Yurkin and A. G. Hoekstra, J. Quant. Spectrosc. Radiat. Transfer 106, 558 (2007).

[6] M. A. Yurkin and M. Kahnert, J. Quant. Spectrosc. Radiat. Transfer 123, 176 (2013).

[7] J. Liu, H. Cai, L. Kong, and X. Zhu, Mater. Sci. Technol. 31, 857 (2015).

[8] M. A. Yurkin, K. A. Semyanov, V. P. Maltsev, and A. G. Hoekstra, Opt. Express 15, 16561 (2007).

[9] J. G. Van Bladel, Electromagnetic Fields, 2nd ed. (Wiley, Hoboken, N.J., 2007).

[10] M. I. Sancer, K. Sertel, J. L. Volakis, and P. V. Alstine, IEEE Trans. Antennas Propag. 54, 1488 (2006).

[11] A. D. Yaghjian, IEEE Proc. 68, 248 (1980).

[12] A. Kirsch, Inv. Probl. Imag. 1, 159 (2007).

[13] M. C. van Beurden and S. J. L. van Eijndhoven, J. Eng. Math. 62, 289 (2008).

[14] M. Costabel, E. Darrigrand, and E. H. Koné, J. Comput. Appl. Math. 234, 1817 (2010).

[15] N. V. Budko and A. B. Samokhin, SIAM J. Sci. Comput. 28, 682 (2006).

[16] D. S. Jones, The Theory of Electromagnetism (Pergamon Press, Oxford, 1964).

[17] M. I. Mishchenko, Opt. Express 15, 13188 (2007).

[18] C. H. Wilcox, Commun. Pure Appl. Math. 9, 115 (1956).

[19] D. A. Smunev, P. C. Chaumet, and M. A. Yurkin, J. Quant. Spectrosc. Radiat. Transfer 156, 67 (2015).

[20] A. D. Yaghjian, A Direct Approach to the Derivation of Electric Dyadic Green's Functions (Nat. Bur. Stand., 1978).

[21] D. Colton and R. Kress, Inverse Acoustic and Electromagnetic Scattering Theory, 3rd ed. (Springer, New York, 2013).

[22] J. Markkanen, Radio Sci. 52, 1301 (2017).

[23] J. Rahola, SIAM J. Sci. Comput. 21, 1740 (2000). 
[24] M. Costabel, E. Darrigrand, and H. Sakly, C. R. Acad. Sci. Paris, Ser. I 350, 193 (2012).

[25] J. Markkanen and P. Ylä-Oijala, J. Quant. Spectrosc. Radiat. Transfer 178, 269 (2016).

[26] N. V. Budko, A. B. Samokhin, and A. A. Samokhin, Diff. Equat. 41, 1262 (2005).

[27] S. S. Hill and R. E. Benner, in Optical Effects Associated with Small Particles, edited by R. K. Chang and P. W. Barber (World Scientific, Singapore, 1988), pp. 1-61.

[28] M. Gastine, L. Courtois, and J. L. Dormann, IEEE Trans. Microw. Theory Tech. 15, 694 (1967).

[29] V. A. Markel, J. Nanophoton. 4, 041555 (2010).

[30] P. R. Conwell, P. W. Barber, and C. K. Rushforth, J. Opt. Soc. Am. A 1, 62 (1984).

[31] C. C. Lam, P. T. Leung, and K. Young, J. Opt. Soc. Am. B 9, 1585 (1992).

[32] B. A. Hunter, M. A. Box, and B. Maier, J. Opt. Soc. Am. A 5, 1281 (1988).

[33] B. R. Johnson, J. Opt. Soc. Am. A 10, 343 (1993).

[34] G. Videen, J. Li, and P. Chýlek, J. Opt. Soc. Am. A 12, 916 (1995).

[35] R. Fuchs and K. L. Kliewer, J. Opt. Soc. Am. 58, 319 (1968).

[36] D. S. Jones, Quart. J. Mech. Appl. Math. 50, 499 (1997).

[37] P. Yla-Oijala, S. P. Kiminki, J. Markkanen, and S. Jarvenpaa, IEEE Antennas Propag. Mag. 55, 310 (2013).

[38] M. Kline, Commun. Pure Appl. Math. 4, 225 (1951).

[39] A. Kirsch and F. Hettlich, The Mathematical Theory of Time-Harmonic Maxwell's Equations: Expansion, Integral, and Variational Methods (Springer, New York, 2014).

[40] M. I. Mishchenko, J. M. Dlugach, M. A. Yurkin, L. Bi, B. Cairns, L. Liu, R. L. Panetta, L. D. Travis, P. Yang, and N. T. Zakharova, Phys. Rep. 632, 1 (2016).

[41] M. A. Yurkin, M. Min, and A. G. Hoekstra, Phys. Rev. E 82, 036703 (2010). 